\title{
Study of the Electrochemical Behavior of Merocyanine and Merocarbocyanine Salts and Their Transformation into ח-Electron Donor Molecules, Namely Tetrathiatetraazafulvalenes
}

\author{
Manel Khiat ${ }^{1^{*}}$, Fatima-Zohra Zradni ${ }^{1}$, Souad Kasmi-Mir ${ }^{2}$, and Alejandro Baeza ${ }^{3}$ \\ ${ }^{1}$ Laboratory of Organic Synthesis, Physico-Chemistry, Biomolecules and Environment (LSPBE), Department of Chemical \\ Engineering, Faculty of Chemistry, University of Sciences and Technology of Oran "Mohamed Boudiaf"- USTOMB, El Mnaouar \\ BP 1505, Bir El Djir 31000 Oran, Algeria \\ ${ }^{2}$ Faculty of Science, Department of Chemistry, Saad Dahlab University Blida 1, 9000 Blida, Algeria \\ ${ }^{3}$ Institute of Organic Synthesis (ISO), Faculty of Sciences, University of Alicante Carretera of San Vicente del Raspeig, s/n, 03690 \\ Alicante, Spain
}

* Corresponding author:

tel: $+213-662135178$

email:t_khiatmanel@yahoo.fr

Received: July 26, 2020

Accepted: December 28, 2020

DOI: $10.22146 / \mathrm{ijc} .58132$

\begin{abstract}
An electrochemical study using the cyclic voltammetry method was carried out on some previously prepared merocyanines salts, namely thiazolideniumsulfonate salts $\mathbf{5} \boldsymbol{a}-\boldsymbol{b}$, and thiazolidenium chloride salts $\mathbf{6} \boldsymbol{a}-\boldsymbol{b}$, and merocarbocyanines salts, namely alkylidenthiazolidenium sulfonate salt $\mathbf{5 c}$, and alkylidenthiazolidenium chloride salt $\mathbf{6} \boldsymbol{c}$. These salts are transformed by dimerization in situ in a voltammetric cell into tetrathiatetraazafulvalenes (TTTAFs) 7a-b, 7'a-b, 8c, and $\mathbf{8}^{\prime} \boldsymbol{c}$ supposed to be $\pi$-electron donor molecules due to the existing conjugation in their structure. The structure of all new chemically synthesized molecules was confirmed by $I R,{ }^{1} \mathrm{H}-\mathrm{NMR},{ }^{13} \mathrm{C}-\mathrm{NMR}$, and MS. The transformation of salts into TTTAF was confirmed by a reversible voltammogram curve and the variation of observed potentials.
\end{abstract}

Keywords: rhodanines; thiazolium salts; merocyanines; tetrathiafulvalenes; dithiadiazafulvalenes; cyclic voltammetry

\section{- INTRODUCTION}

For many years, organic materials have been considered as insulators. However, the discovery of perylene bromide in 1954 as a semi-conductor profoundly upset this vision by showing that organic materials could possess electrical conduction properties [1]. In a few decades, the development and study of organic conductors and superconductors have occupied a considerable position in the molecular sciences [2].

One of the first breakthroughs in the field of organic electrical conductors came with the discovering of the TTF (Fig. 1), endowed with a $\pi$-electron donor character [3], and TCNQ (Fig. 1) (structure b), endowed with a $\pi$ electron acceptor character [4]. The association of both motifs leads to the formation of the crystalline charge transfer complex TTF-TCNQ, which was the first organic compound with extraordinary electrical properties [5].
After this pioneering work, the synthesis of many other charge-transfer complexes by modifications made on the TTF structure has been developed. For example, the substitution of sulfur by other chalcogens such as tellurium and selenium, as in the case of tetramethyltetraselenafulvalene (TMTSeF), is called radical ionic salts of Bechgaard [6].

In addition, these TTF derivatives have been the object of many studies because of their various applications such as Self-Assembled Monolayer (SAM) systems functionalized with TTF [7-10], fructose biosensors (TTF co-immobilized on SAM) [11], metal ion sensors (complexation of crown ethers to TTF-SAM) [12-18], complexes of C60-tetrathiafulvalene [19-24], supramolecular switches [25], natural photosynthesis structures (TTF containing catenanes) [26], their use in enzymatic reactions [27-28], radical reactions [29-30], 
<smiles>CC1=C(C)SC(=C2SC(C)=C(C)S2)S1</smiles>

TTF

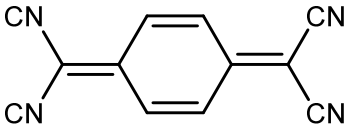

TCNQ

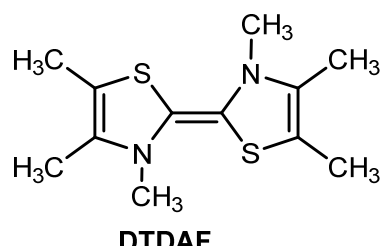

DTDAF

Fig 1. Structure of tetrathiafulvalene (TTF), tetracyanoquinodimethane (TCNQ) and dithiadiazafulvalene (DTDAF)

redox polymers [31-32], dendritic macromolecules incorporating TTF [33-34]. Therefore, it is not surprising that considerable efforts are being made to synthesize and study new electron-donating organic molecules, allowing synergy between physical properties, such as electrical conductivity or superconductivity and magnetic effects, optical properties or spin transition [35]. Indeed a nitrogenous derivative of the TTF has been synthesized by substituting the sulfur atoms with nitrogen ones, namely the dithiadiazafulvalenes (DTDAFs) (Fig. 1) [36].

The idea behind this work is to obtain electroactive species, analogs to TTFs, and DTDAFs, namely tetrathiatetraazafulvalenes (TTTAFs) 7a-b, 7'a-b, 8c, and $\mathbf{8}$ 'c, by dimerization of merocyanine salts in situ in an electrochemical cell. Indeed, the electrochemical studies using the cyclic Voltammetry method have been performed on thiazolidenium sulfonate salts $\mathbf{5 a - b}$, thiazolidenium chloride salts $\mathbf{6 a - b}$, alkylidenthiazolidenium sulfonate salt $\mathbf{5 c}$, and alkylidenthiazolidenium chloride salt $\mathbf{6 c}$ as precursor candidates to demonstrate their redox behavior, their cathodic coupling, and consequently their transformation into TTTAFs.

\section{- EXPERIMENTAL SECTION}

\section{Materials}

The chemicals and reagents used for the synthesis were purchased at $99 \%$ purity from commercial sources (Biochem Chemopharma and Sigma-Aldrich companies in France).

\section{Instrumentation}

The conversion of the reactions and purity of the synthesized compounds was verified by thin-layer chromatography (TLC) on silica gel G. The melting points were determined on a Buchi apparatus (melting point B-545). Infrared spectroscopy (IR) was recorded using $\mathrm{KBr}$ pellets, in a Fourier transform spectrophotometer JASCO FT/IR between 400 and $4000 \mathrm{~cm}^{-1}$, NMR spectra were recorded on Bruker Avance 400 spectrometer $\left(400 \mathrm{MHz}\right.$ for ${ }^{1} \mathrm{H}$ and $101 \mathrm{MHz}$ for ${ }^{13} \mathrm{C}$ ), using $\mathrm{CDCl}_{3}, \mathrm{DMSO}-\mathrm{d} 6$ as a solvent. The values of the chemical shifts are expressed in scale delta part per million $(\delta \mathrm{ppm})$ compared to tetramethylsilane (TMS) used as an internal standard. Mass spectra were obtained on the GCMS $\backslash$ QP mass spectrometer at $70 \mathrm{eV}$. The cyclic voltammetry was carried out with Voltalab 40 PGZ 301 potentiostat. The counter electrode was a platinum $(\mathrm{Pt})$ wire, and the reference electrode an $\mathrm{Ag} / \mathrm{AgCl}$ electrode. The working electrode was a platinum disk $\left(\mathrm{A}=1 \mathrm{~mm}^{2}\right)$.

\section{Procedure}

\section{General procedure for the synthesis of thioxothiazolidinones (1a-b) and alkylidenethioxotiazolidinone (1c)}

Under magnetic stirring at $0{ }^{\circ} \mathrm{C}$, in a $250 \mathrm{~mL}$ round-bottom flask, $0.1 \mathrm{~mol}$ of primary amine $\left(\mathrm{RNH}_{2}\right)$ and $0.18 \mathrm{~mol}(20 \mathrm{~mL})$ of concentrated ammonium hydroxide $\left(\mathrm{NH}_{4} \mathrm{OH}\right)$ were mixed for $15 \mathrm{~min}$. Then $0.1 \mathrm{~mol}(7 \mathrm{~mL})$ of carbon disulfide $\left(\mathrm{CS}_{2}\right)$ was added dropwise to the mixture. After stirring for $2 \mathrm{~h}$, the dithiocarbamate salt (DTC) formed was filtered and then washed several times with diethyl ether or recovered using a separating funnel if it is a liquid. The DTC prepared was dissolved in $150 \mathrm{~mL}$ of water, and $0.11 \mathrm{~mol}$ (10.6 g) of chloroacetic acid were added in two portions, under magnetic stirring for $2 \mathrm{~h}$ at $0{ }^{\circ} \mathrm{C}$. The reaction mixture is then heated in a bath between 60 $70{ }^{\circ} \mathrm{C}$ for $3 \mathrm{~h}$. After cooling down to room temperature, the precipitate was separated by decantation to give thioxothiazolidinones (1a-b) as crystals and (1c) as a liquid. A water/acetone mixture was added to the 
compound $1 \mathrm{c}$ with stirring and was heated at $60^{\circ} \mathrm{C}$. After cooling down to room temperature, the mixture is left standing for $48 \mathrm{~h}$ to obtain the alkylidenethioxotiazolidinone (3c) in the form of orange crystals.

3-Benzyl-2-thioxo-1,3-thiazolidin-4-one (1a). Beige crystals; Yield: 68\%; M.p.: $74{ }^{\circ} \mathrm{C} ; \mathrm{R}_{\mathrm{f}}$ : 0.80 (ethyl acetate/methanol 6:4). IR ( $\left.\mathrm{KBr}, v \mathrm{~cm}^{-1}\right): 3086.9(\mathrm{C}-\mathrm{H}$ aromatic), 2963.6 (C-H aliphatic), $1698.9(\mathrm{C}=\mathrm{O}), 1582.8$, $1452.2\left(\mathrm{C}=\mathrm{C}\right.$ aromatic), $1164.5(\mathrm{C}=\mathrm{S}) .{ }^{1} \mathrm{H}-\mathrm{NMR}(400$ $\left.\mathrm{MHz}, \mathrm{CDCl}_{3}\right) \delta$ 7.52-7.41 (m, 1H), 7.38-7.26 (m, 2H), $5.20(\mathrm{~s}, 1 \mathrm{H}), 3.97(\mathrm{~s}, 1 \mathrm{H}) .{ }^{13} \mathrm{C}-\mathrm{NMR}\left(101 \mathrm{MHz}, \mathrm{CDCl}_{3}\right) \delta$ 201.08 (-C=S), 173.87 (-C=O), 134.69 (C, Ar), 129.06 (CH, Ar), 128.91(CH, Ar), $128.58(\mathrm{CH}, \mathrm{Ar}), 128.41(\mathrm{CH}$, Ar), $128.21(\mathrm{CH}, \mathrm{Ar}), 127.56(\mathrm{CH}, \mathrm{Ar}), 47.61\left(\mathrm{H}_{2} \mathrm{C}-\mathrm{N}\right)$, $35.42\left(-\mathrm{S}-\mathrm{CH}_{2}\right)$. Mass spectrum, $\mathrm{m} / \mathrm{z}$ : 223.1 .

3-Phenyl-2-thioxo-1,3-thiazolidin-4-one (1b). Clear Yellow Crystals; Yield: 59\%; M.p.: $115^{\circ} \mathrm{C}$; $\mathrm{R}_{\mathrm{f}}$ : 0.35 (ethyl acetate/ methanol 6:4). IR (KBr, $\left.v \mathrm{~cm}^{-1}\right)$ : $3086.9(\mathrm{C}-\mathrm{H}$ aromatic), 2964.3 (C-H aliphatic), 1731.5 (C=O), 1582.5$1452.4\left(\mathrm{C}=\mathrm{C}\right.$ aromatic), $1165.7(\mathrm{C}=\mathrm{S}) .{ }^{1} \mathrm{H}-\mathrm{NMR}(400$ $\left.\mathrm{MHz}, \mathrm{CDCl}_{3}\right) \delta 8.11(\mathrm{~s}, 1 \mathrm{H}), 7.77-7.52(\mathrm{~m}, 1 \mathrm{H}), 7.51-7.34$ (m, 4H), 7.34-7.11 (m, 3H), $4.85(\mathrm{~s}, 1 \mathrm{H}), 4.20(\mathrm{~s}, 1 \mathrm{H}) .{ }^{13} \mathrm{C}-$ NMR (101 MHz, $\left.\mathrm{CDCl}_{3}\right) \delta 201.26(-\mathrm{C}=\mathrm{S}), 173.52$ ($\mathrm{C}=\mathrm{O}), 137.12$ (C, Ar), 130.18 (CH, Ar), $129.79(\mathrm{CH}, \mathrm{Ar})$, $129.65(\mathrm{CH}, \mathrm{Ar}), 129.57(\mathrm{CH}, \mathrm{Ar}), 128.36(\mathrm{CH}, \mathrm{Ar})$, $127.68(\mathrm{CH}, \mathrm{Ar}), 36.45\left(-\mathrm{S}-\mathrm{CH}_{2}\right)$. Mass spectrum, m/z: 209.0.

3-Propyl-2-thioxothiazolidin-4-one (1c). Brown oily liquid; Yield: 62\%; $\mathrm{R}_{\mathrm{f}} 0.78$ (ethyl acetate/methanol 6:4). IR $\left(\mathrm{KBr}, v \mathrm{~cm}^{-1}\right): 2966.5(\mathrm{C}-\mathrm{H}$ aliphatic), $1736.1(\mathrm{C}=\mathrm{O})$, 1187.7 (C=S), $1088.4(\mathrm{C}-\mathrm{N}) .{ }^{1} \mathrm{H}-\mathrm{NMR}\left(400 \mathrm{MHz}, \mathrm{CDCl}_{3}\right)$ $\delta 4.37(\mathrm{~s}, 1 \mathrm{H}), 3.79(\mathrm{~d}, 1 \mathrm{H}), 1.58(\mathrm{~m}, 1 \mathrm{H}), 0.86(\mathrm{t}, 1 \mathrm{H}) .{ }^{13} \mathrm{C}-$ NMR (101 MHz, $\left.\mathrm{CDCl}_{3}\right) \delta 198.32$ (-C=S), 169.81 ($\mathrm{C}=\mathrm{O}), 46.27\left(\mathrm{~N}-\mathrm{CH}_{2}\right), 36.75(-\mathrm{S}-\mathrm{C}-), 21.54\left(\mathrm{H}_{2} \mathrm{C}-\mathrm{C}-\right)$, $11.28\left(\mathrm{CH}_{3}\right)$. Mass spectrum, $\mathrm{m} / \mathrm{z}: 215.1$.

\section{5-(Propan-2-ylidene)-3-propyl-2-thioxo-1,3-}

thiazolidin-4-one (3c). Orange crystals; Yield: 59\%; M.p.: $33^{\circ} \mathrm{C}$; $\mathrm{R}_{\mathrm{f}}: 0.81$ (ethyl acetate/methanol 6:4). IR (KBr, $v \mathrm{~cm}^{-1}$ ): 3084.32 (C-H aliphatic), $1783.15(\mathrm{C}=\mathrm{O}), 1635.93$ $(\mathrm{C}=\mathrm{C}), 1157.40(\mathrm{C}=\mathrm{S}) .{ }^{1} \mathrm{H}-\mathrm{NMR}\left(400 \mathrm{MHz}, \mathrm{CDCl}_{3}\right) \delta 4.01$ (d, 2H), 2.45 (s, 3H), $2.02(\mathrm{~s}, 3 \mathrm{H}), 1.75-1.65(\mathrm{~m}, 2 \mathrm{H}), 0.95$ (t, 3H). ${ }^{13} \mathrm{C}-\mathrm{NMR}\left(101 \mathrm{MHz}, \mathrm{CDCl}_{3}\right) \delta 192.99$ (-C=S),
165.37 (-C=O), 149.76 (-C=C-S-), 120.99 (=C-S-), $45.84\left(\mathrm{~N}-\mathrm{CH}_{2}\right), 21.70\left(\mathrm{H}_{2} \mathrm{C}-\mathrm{CH}_{2}-\right), 20.35\left(\mathrm{H}_{3} \mathrm{C}-\mathrm{C}=\mathrm{C}\right)$, $11.26\left(\mathrm{CH}_{3}\right)$. Mass spectrum, m/z: 215.1 .

\section{General procedure for the synthesis of thiazolinethiones (2a-c)}

In a $250 \mathrm{~mL}$ round-bottom flask, $0.18 \mathrm{~mol}$ of concentrated ammonium hydroxide was mixed with 0.1 mol of a primary amine $\left(\mathrm{RNH}_{2}\right)$ under magnetic stirring at $0{ }^{\circ} \mathrm{C}$. After $15 \mathrm{~min}, 0.1 \mathrm{~mol}$ of carbon disulfide was added dropwise to the mixture, and the stirring was maintained for $2 \mathrm{~h}$. The dithiocarbamate salt formed was filtered and then washed several times with diethyl ether or recovered using a separating funnel if it is a liquid. The DTC prepared was dissolved in $200 \mathrm{~mL}$ of water. Chloroacetone $(0.15 \mathrm{~mol})$ was added in two portions. The $\mathrm{pH}$ must be acidic ( 2 to 3 ). The reaction mixture was then heated in a water bath at $60-70{ }^{\circ} \mathrm{C}$ for $2 \mathrm{~h}$. The precipitate was separated by filtration and recrystallized from ethanol.

3-Benzyl-4-methyl-1,3-thiazole-2(3H)-thione (2a). Beige crystals; Yield: 83.55\%; M.p.: $91{ }^{\circ} \mathrm{C}$; $\mathrm{R}_{\mathrm{f}}: 0.76$ (Dichloromethane/Methanol 6:4). IR ( $\left.\mathrm{KBr}, v \mathrm{~cm}^{-1}\right)$ : 3075.1 (C-H aromatic), 2956.6 (C-H aliphatic), 1635.0 (C=C aliphatic), 1488.6-1404.6 ( $\mathrm{C}=\mathrm{C}$ aromatic), 1166.4 $(\mathrm{C}=\mathrm{S}) .{ }^{1} \mathrm{H}-\mathrm{NMR}\left(400 \mathrm{MHz}, \mathrm{CDCl}_{3}\right) \delta 7.39-7.26(\mathrm{~m}, 3 \mathrm{H})$, 7.24-7.16 (m, 2H), $6.28(\mathrm{~d}, 1 \mathrm{H}), 5.53(\mathrm{~s}, 2 \mathrm{H}), 2.15(\mathrm{~d}$, $3 \mathrm{H}) .{ }^{13} \mathrm{C}-\mathrm{NMR}\left(101 \mathrm{MHz}, \mathrm{CDCl}_{3}\right) \delta 189.26(-\mathrm{C}=\mathrm{S})$, $140.13(\mathrm{~N}-\mathrm{C}=\mathrm{C}), 134.84$ (C, Ar), 128.91 (CH, Ar), $127.85(\mathrm{CH}, \mathrm{Ar}), 126.64(\mathrm{CH}, \mathrm{Ar}), 106.17(\mathrm{~S}-\mathrm{HC}=\mathrm{C})$, $50.11\left(\mathrm{H}_{2} \mathrm{C}-\mathrm{N}\right), 15.60\left(\mathrm{CH}_{3}\right)$. Mass spectrum, m/z: 221.1. 4-Methyl-3-phenyl-1,3-thiazole-2(3H)-thione (2b). Beige crystals; Yield: $61.55 \%$; M.p.: $124{ }^{\circ} \mathrm{C}$; $\mathrm{R}_{\mathrm{f}}: 0.82$ (Dichloromethane/Methanol 6:4). IR ( $\left.\mathrm{KBr}, v \mathrm{~cm}^{-1}\right)$ : 3097.5 (C-H aromatic), 2957.2 (C-H aliphatic), 1635.1 ( $\mathrm{C}=\mathrm{C}$ aliphatic), $1486.4(\mathrm{C}=\mathrm{C}$ aromatic), $1225.6(\mathrm{C}=\mathrm{S})$. ${ }^{1} \mathrm{H}-\mathrm{NMR}\left(400 \mathrm{MHz}, \mathrm{CDCl}_{3}\right) \delta$ 7.65-7.48 (m, 1H), 7.29$7.19(\mathrm{~m}, 1 \mathrm{H}), 6.36(\mathrm{q}, 1 \mathrm{H}), 1.96(\mathrm{~d}, 1 \mathrm{H}) .{ }^{13} \mathrm{C}-\mathrm{NMR}(101$ $\left.\mathrm{MHz}, \mathrm{CDCl}_{3}\right) \delta 190.17(-\mathrm{C}=\mathrm{S}), 140.19(\mathrm{~N}-\mathrm{C}=\mathrm{C}), 137.79$ (C, Ar), $129.97(\mathrm{CH}, \mathrm{Ar}), 129.70(\mathrm{CH}, \mathrm{Ar}), 128.16(\mathrm{CH}$, Ar), $106.42(\mathrm{~S}-\mathrm{HC}=\mathrm{C}), 16.19\left(\mathrm{CH}_{3}\right)$. Mass spectrum, $\mathrm{m} / \mathrm{z}: 206.1$.

4-Methyl-3-propyl-1,3-thiazole-2(3H)-thione (2c). Beige crystals; Yield: 76.52\%; M.p.: $83{ }^{\circ} \mathrm{C}$; $\mathrm{R}_{\mathrm{f}}: 0.65$ 
(Dichloromethane/Methanol 6:4). IR (KBr, $\left.v \mathrm{~cm}^{-1}\right)$ : 3093.9 (C-H aromatic), 2944.9 (C-H aliphatic), 1634.5 (C=C aliphatic), 1226.8 ( $\mathrm{C}=\mathrm{S}) .{ }^{1} \mathrm{H}-\mathrm{NMR}(400 \mathrm{MHz}$, $\left.\mathrm{CDCl}_{3}\right) \delta 6.26(\mathrm{~d}, 1 \mathrm{H}), 4.16-4.06(\mathrm{~m}, 1 \mathrm{H}), 2.29(\mathrm{~d}, 1 \mathrm{H})$, $1.86-1.71(\mathrm{~m}, 1 \mathrm{H}), 0.99(\mathrm{t}, 1 \mathrm{H}) .{ }^{13} \mathrm{C}-\mathrm{NMR}(101 \mathrm{MHz}$, $\left.\mathrm{CDCl}_{3}\right) \delta 187.85(-\mathrm{C}=\mathrm{S}), 139.59(\mathrm{~N}-\mathrm{C}=\mathrm{C}), 106.31 \quad(\mathrm{~S}-$ $\mathrm{HC}=\mathrm{C}), 48.77\left(\mathrm{H}_{2} \mathrm{C}-\mathrm{N}\right), 20.98\left(\mathrm{CH}_{2}\right), 15.43\left(\mathrm{CH}_{3}\right), 11.21$ $\left(\mathrm{CH}_{3}\right)$. Mass spectrum, $\mathrm{m} / \mathrm{z}: 173.1$.

\section{General procedure for the synthesis of thiazolium salts (3a-b)}

In a flask containing $100 \mathrm{~mL}$ of acetone, $5 \mathrm{mmol}$ of thiazolinethione already prepared was mixed with $15 \mathrm{mmol}$ of methyl iodide (MeI). The mixture was stirred at room temperature for $24 \mathrm{~h}$. The salt obtained was filtered and then washed with acetone.

\section{3-Benzyl-4-methyl-2-(methylsulfanyl)-1,3-thiazol-3-} ium-iodide (3a). White crystals; Yield: 60.58\%; M.p.: $139^{\circ} \mathrm{C}$; $\mathrm{R}_{\mathrm{f}}$ 0.71. IR (KBr, $\left.v \mathrm{~cm}^{-1}\right): 3026.7$ (C-H aromatic), 2949.1 (C-H aliphatic), 1634.8 (C=C aliphatic), 1491.21405.8 (C=C aromatic), 1077 (C-S). ${ }^{1} \mathrm{H}-\mathrm{NMR}(400 \mathrm{MHz}$, DMSO) $\delta 7.93(\mathrm{~s}, 1 \mathrm{H}), 7.45-7.33(\mathrm{~m}, 9 \mathrm{H}), 7.27-7.09(\mathrm{~m}$, $5 \mathrm{H}), 5.61(\mathrm{~s}, 5 \mathrm{H}), 2.99(\mathrm{~s}, 7 \mathrm{H}), 2.53-2.50(\mathrm{~m}, 4 \mathrm{H}), 2.45(\mathrm{~d}$, $7 \mathrm{H}) .{ }^{13} \mathrm{C}-\mathrm{NMR}(101 \mathrm{MHz}, \mathrm{DMSO}) \delta 177.16(-\mathrm{C}=\mathrm{S})$, $146.61(\mathrm{~N}-\mathrm{C}=\mathrm{C}), 132.61$ (C, Ar), $129.63(\mathrm{CH}, \mathrm{Ar}), 129.00$ $(\mathrm{CH}, \mathrm{Ar}), 127.13(\mathrm{CH}, \mathrm{Ar}), 118.24(\mathrm{~S}-\mathrm{HC}=\mathrm{C}), 53.49$ $\left(\mathrm{H}_{2} \mathrm{C}-\mathrm{N}\right), 18.54\left(-\mathrm{S}-\mathrm{CH}_{3}\right), 14.36\left(\mathrm{CH}_{3}\right)$.

4-Methyl-2-(methylsulfanyl)-3-phenyl-1,3-thiazol-3ium-iodide (3b). Beige crystals; Yield: 53.64\%; M.p.: $132{ }^{\circ} \mathrm{C} ; \mathrm{R}_{\mathrm{f}}$ 0.78.IR ( $\left.\mathrm{KBr}, v \mathrm{~cm}^{-1}\right): 3095.6$ (C-H aromatic), 2944.8 (C-H aliphatic), 1634.0 (C=C aliphatic), 1484.1 (C=C aromatic), 1077 (C-S). ${ }^{1} \mathrm{H}-\mathrm{NMR}(400 \mathrm{MHz}$, DMSO) $\delta 7.99(\mathrm{~d}, 1 \mathrm{H}), 7.82-7.63(\mathrm{~m}, 5 \mathrm{H}), 3.74(\mathrm{~s}, 1 \mathrm{H})$, $2.91(\mathrm{~s}, 3 \mathrm{H}), 2.16(\mathrm{~d}, 3 \mathrm{H}) .{ }^{13} \mathrm{C}-\mathrm{NMR}(101 \mathrm{MHz}, \mathrm{DMSO}) \delta$ $179.02(-\mathrm{C}=\mathrm{S}), 146.36$ (N-C=C), 135.19 (C, Ar), 132.43 $(\mathrm{CH}, \mathrm{Ar}), 131.28(\mathrm{CH}, \mathrm{Ar}), 127.35(\mathrm{CH}, \mathrm{Ar}), 117.73$ (S$\mathrm{HC}=\mathrm{C}), 18.30\left(-\mathrm{S}-\mathrm{CH}_{3}\right), 14.60\left(\mathrm{CH}_{3}\right)$.

\section{General procedure for the synthesis of merocyanines (4a-b) and merocarbocyanines (4c)}

$1^{\text {st }}$ Method: In a flask containing $20 \mathrm{~mL}$ of acetone and under magnetic stirring, $10 \mathrm{mmol}$ of thiazolium salt (3a-b) was mixed with $10 \mathrm{mmol}$ of rhodanine (1a-b) or arylidenerhodanine (3c) already prepared, and $2 \mathrm{~mL}$ of triethylamine was added to the mixture. After stirring for
$24 \mathrm{~h}$ at room temperature or $1 \mathrm{~h}$ at reflux, the precipitate was separated by filtration and recrystallized from a mixture of Ethanol/ DMF).

$2^{\text {nd }}$ Method: In a flask containing $20 \mathrm{~mL}$ of acetonitrile and under magnetic stirring, $10 \mathrm{mmol}$ of thiazolinethione (2a-c) was mixed with $15 \mathrm{mmol}$ of dimethyl sulfate. After heating for $1 \mathrm{~h}$ and cooling back to room temperature, $10 \mathrm{mmol}$ of rhodanine $(\mathbf{1 a}-\mathbf{b})$ or arylidenerhodanine (3c) and $2 \mathrm{~mL}$ of triethylamine were added. Immediate formation of a solid was noticed. The reaction is left for $1 \mathrm{~h}$ at reflux until the release of $\mathrm{CH}_{3} \mathrm{SH}$. The resulting solid was filtered and recrystallized from a mixture (Ethanol/DMF).

(5E)-3-Benzyl-5-(3-benzyl-4-methyl-1,3-thiazol-2(3H) -ylidene)-2-thioxo-1,3-thiazolidin-4-one (4a). Yellow crystals; Yield: $84.21 \%$; M.p.: $191{ }^{\circ} \mathrm{C}$; $\mathrm{R}_{\mathrm{f}}: 0.30$ (ethyl acetate/methanol 6:4). IR (KBr, $\left.v \mathrm{~cm}^{-1}\right): 3078.2(\mathrm{C}-\mathrm{H}$ aromatic), 2958.4 (C-H aliphatic), $1891.6(\mathrm{C}=\mathrm{O}), 1624.3$ ( $\mathrm{C}=\mathrm{C}$ aliphatic), 1597.2-1429.6 ( $\mathrm{C}=\mathrm{C}$ aromatic), 1192.7 $(\mathrm{C}=\mathrm{S}) .{ }^{1} \mathrm{H}-\mathrm{NMR}\left(400 \mathrm{MHz}, \mathrm{CDCl}_{3}\right) \delta$ 7.59-7.50 $(\mathrm{m}, 1 \mathrm{H})$, 7.42-7.33 (m, 1H), 7.32-7.18 (m, 1H), $7.02(\mathrm{~d}, 1 \mathrm{H}), 6.39$ (d, $1 \mathrm{H}), 5.33(\mathrm{~d}, 1 \mathrm{H}),{ }^{13} \mathrm{C}-\mathrm{NMR}\left(101 \mathrm{MHz}, \mathrm{CDCl}_{3}\right) \delta$ $186.80 \quad(-\mathrm{C}=\mathrm{S}), 165.16 \quad(-\mathrm{C}=\mathrm{O}), 156.94 \quad(-\mathrm{S}-\mathrm{C}=\mathrm{C})$, 137.72 (C, Ar), 135.77 (C, Ar), 133.91 (CH, Ar), 129.49 $(\mathrm{CH}, \mathrm{Ar}), 129.03(\mathrm{CH}, \mathrm{Ar}), 128.51(\mathrm{CH}, \mathrm{Ar}), 128.34(\mathrm{CH}$, $\mathrm{Ar}), 127.63(\mathrm{CH}, \mathrm{Ar}), 125.25(\mathrm{~N}-\mathrm{C}=\mathrm{C}), 105.29$ (S$\mathrm{HC}=\mathrm{C}), 82.40(-\mathrm{C}=\mathrm{C}-\mathrm{S}-), 50.62\left(\mathrm{~N}-\mathrm{CH}_{2}-\mathrm{C}=\mathrm{C}\right), 47.74$ $\left(\mathrm{N}-\mathrm{CH}_{2}-\mathrm{C}=\mathrm{C}\right), 14.10\left(\mathrm{CH}_{3}\right)$.

(5E)-5-(4-Methyl-3-phenyl-1,3-thiazol-2(3H)-ylidene)3-phenyl-2-thioxo-1,3-thiazolidin-4-one (4b). Yellow crystals; Yield: $81.53 \%$; M.p.: $235{ }^{\circ} \mathrm{C}$; $\mathrm{R}_{\mathrm{f}}: 0.27$ (ethyl acetate/methanol 6:4). IR ( $\left.\mathrm{KBr}, v \mathrm{~cm}^{-1}\right): 3078.2$ (C-H aromatic), 2959.6 (C-H aliphatic), $1883.3(\mathrm{C}=\mathrm{O}$ ), 1634.3 ( $\mathrm{C}=\mathrm{C}$ aliphatic), 1591.0-1482.8 ( $\mathrm{C}=\mathrm{C}$ aromatic), $1228.4(\mathrm{C}=\mathrm{S}) .{ }^{1} \mathrm{H}-\mathrm{NMR}\left(400 \mathrm{MHz}, \mathrm{CDCl}_{3}\right) \delta 7.77-7.68$ (m, 1H), $7.65(\mathrm{t}, 2 \mathrm{H}), 7.55-7.48(\mathrm{~m}, 2 \mathrm{H}), 7.47-7.42(\mathrm{~m}$, $1 \mathrm{H}), 7.39-7.35(\mathrm{~m}, 2 \mathrm{H}), 7.31-7.26(\mathrm{~m}, 2 \mathrm{H}), 6.39(\mathrm{~d}, 1 \mathrm{H})$, $1.95(\mathrm{~d}, 3 \mathrm{H}) .{ }^{13} \mathrm{C}-\mathrm{NMR}\left(101 \mathrm{MHz}, \mathrm{CDCl}_{3}\right) \delta 189.07(-$ $\mathrm{C}=\mathrm{S}), 165.45(-\mathrm{C}=\mathrm{O}), 155.74(-\mathrm{S}-\mathrm{C}=\mathrm{C}), 137.56(\mathrm{~N}-$ $\mathrm{C}=\mathrm{C}$ ), 136.14 (C, Ar), 135.07 (C, Ar), 131.79 (CH, Ar), $130.62(\mathrm{CH}, \mathrm{Ar}), 129.28(\mathrm{CH}, \mathrm{Ar}), 129.26(\mathrm{CH}, \mathrm{Ar})$, $129.04(\mathrm{CH}, \mathrm{Ar}), 128.43(\mathrm{CH}, \mathrm{Ar}), 104.34(-\mathrm{S}-\mathrm{C}=\mathrm{C})$, $84.68(-\mathrm{S}-\mathrm{C}=\mathrm{C}), 14.49\left(\mathrm{CH}_{3}\right)$. 
(E)-5-((E)-1-(4-Methyl-3-propylthiazol-2(3H)-ylidene) propan-2-ylidene)-3-propyl-2-thioxothiazolidin-4-

one (4c). Purple crystals; Yield: 87.32\%; M.p.: $149^{\circ} \mathrm{C}$; $\mathrm{R}_{\mathrm{f}}$. 0.64 (ethyl acetate/methanol 6:4). IR $\left(\mathrm{KBr}, v \mathrm{~cm}^{-1}\right)$ : 2964.32 (C-H aliphatic), 2876.73 (C-H aliphatic), $1704.83(\mathrm{C}=\mathrm{O}), 1615.61(\mathrm{C}=\mathrm{C}$ aliphatic), $1150.47(\mathrm{C}=\mathrm{S})$. ${ }^{1} \mathrm{H}-\mathrm{NMR}\left(400 \mathrm{MHz}, \mathrm{CDCl}_{3}\right) \delta 8.06(\mathrm{~s}, 1 \mathrm{H}), 7.28(\mathrm{~s}, 1 \mathrm{H})$, $6.20(\mathrm{~s}, 1 \mathrm{H}), 5.27(\mathrm{~d}, 1 \mathrm{H}), 4.49-3.68(\mathrm{~m}, 5 \mathrm{H}), 3.29-1.59$ (m, $14 \mathrm{H}), 1.38-0.75(\mathrm{~m}, 8 \mathrm{H}), 0.37-0.17(\mathrm{~m}, 1 \mathrm{H}) .{ }^{13} \mathrm{C}-$ NMR $\left(101 \mathrm{MHz}, \mathrm{CDCl}_{3}\right) \delta 187.79(-\mathrm{C}=\mathrm{S}), 163.45(-$ $\mathrm{C}=\mathrm{O}), 160.01 \quad(\mathrm{C}=\mathrm{C}-\mathrm{C}), 146.80 \quad(\mathrm{~N}-\mathrm{C}=\mathrm{C}), 138.90(\mathrm{~N}-$ $\mathrm{C}=\mathrm{C}), 102.28$ (S-C=), $96.03(=\mathrm{C}-\mathrm{H}), 48.39\left(\mathrm{~N}-\mathrm{CH}_{2}\right)$, $45.88 \quad\left(\mathrm{~N}-\mathrm{CH}_{2}\right), \quad 23.02 \quad\left(\mathrm{CH}_{2}\right), \quad 20.62 \quad\left(\mathrm{CH}_{2}\right)$, $20.48\left(\mathrm{CH}_{3}\right), 14.74\left(\mathrm{CH}_{3}\right), 11.42\left(\mathrm{CH}_{3}\right), 11.3\left(\mathrm{CH}_{3}\right)$.

Note: when the merocyanines (4a-b) and merocarbocyanines (4c) were injected into the mass spectroscopy apparatus, they decompose because of their instability.

\section{General procedure for the synthesis of thiazolidenium sulfonate salts (5a-b) and alkylidenthiazolidenium sulfonate salt (5c)}

A mixture of $5 \mathrm{mmol}$ of thiazolydenethioxothiazoli dinones or alkylidenethiazolydene-thioxothiazolidinone, 15 mmoles of methylparatoluenesulphonate (MPTS), and $5 \mathrm{~mL}$ of DMF is stirred at $110-120^{\circ} \mathrm{C}$ for $4 \mathrm{~h}$. After this time, the reaction mixture is cooled down to $40^{\circ} \mathrm{C}$, and then $50 \mathrm{~mL}$ of acetone were added. When the reaction is complete, the mixture is left to reach room temperature and then refrigerated overnight. The corresponding salt obtained is filtered and dried under a vacuum.

(E)-3,3'-Dibenzyl-4-methyl-2'-(methylthio)-4'-oxo-3H, 4'H-[2,5'-bithiazolylidene]-3'-ium-4-methylbenzene sulfonate (5a). Brown crystals; Yield: 78.96\%; M.p.: 239 ${ }^{\circ} \mathrm{C}$. ${ }^{1} \mathrm{H}-\mathrm{NMR}(400 \mathrm{MHz}, \mathrm{DMSO}) \delta 8.12-7.96(\mathrm{~m}, 2 \mathrm{H})$, 7.75-7.69 (m, 2H), 7.64-7.60 (m, 1H), 7.51-7.42 (m, 1H), 7.38-7.33 (m, 2H), 7.29-7.26 (m, 3H), 7.24 (m, 1H), 7.20$7.18(\mathrm{~m}, 2 \mathrm{H}), 5.98(\mathrm{~d}, 1 \mathrm{H}), 4.49(\mathrm{~d}, 2 \mathrm{H}), 3.52(\mathrm{~d}, 2 \mathrm{H}), 2.73$ (s, 3H), $2.61(\mathrm{~d}, 3 \mathrm{H}), 2.14(\mathrm{~d}, 3 \mathrm{H}) \cdot{ }^{13} \mathrm{C}-\mathrm{NMR}(101 \mathrm{MHz}$, DMSO) $\delta 174.21(-\mathrm{C}=\mathrm{O}), 160.14(\mathrm{C}=\mathrm{N}), 149.27(\mathrm{C}, \mathrm{Ar}-$ $\mathrm{S}=\mathrm{O}$ ), 140.02 (C, Ar), 138.73 (C, Ar), 136.94 (C, Ar), $131.58(\mathrm{CH}, \mathrm{Ar}), 131.20(\mathrm{CH}, \mathrm{Ar}), 130.98(\mathrm{CH}, \mathrm{Ar})$, $130.64(\mathrm{CH}, \mathrm{Ar}), 129.86(\mathrm{CH}, \mathrm{Ar}), 128.52(\mathrm{CH}, \mathrm{Ar})$, $126.09(\mathrm{CH}, \mathrm{Ar}), 117.20(\mathrm{~N}-\mathrm{C}=\mathrm{C}), 108.87(\mathrm{~S}-\mathrm{C}=\mathrm{C})$,
$105.78(\mathrm{~S}-\mathrm{C}=\mathrm{C}), 52.73\left(\mathrm{~N}-\mathrm{CH}_{2}-\mathrm{C}=\mathrm{C}\right), 49.63\left(\mathrm{~N}-\mathrm{CH}_{2}-\right.$ $\mathrm{C}=\mathrm{C}), 22.06\left(\mathrm{CH}_{3}\right), 15.37\left(\mathrm{CH}_{3}\right), 14.25\left(\mathrm{CH}_{3}\right)$.

(E)-4-Methyl-2'-(methylthio)-4'-oxo-3,3'-diphenyl3H,4'H-[2,5'-bithiazolylidene]-3'-ium-4-methylben zenesulfonate (5b). Brown crystals; Yield: 71.24\%; M.p.: $256{ }^{\circ} \mathrm{C} .{ }^{1} \mathrm{H}-\mathrm{NMR}(400 \mathrm{MHz}, \mathrm{DMSO}) \delta 10.65-$ $10.58(\mathrm{~m}, 2 \mathrm{H}), 8.29-8.17(\mathrm{~m}, 2 \mathrm{H}), 7.96(\mathrm{~m}, 2 \mathrm{H}), 7.58(\mathrm{~m}$, $2 \mathrm{H}), 7.89-7.78(\mathrm{~m}, 1 \mathrm{H}), 7.46-7.42(\mathrm{~m}, 2 \mathrm{H}), 7.37-7.30$ (m, 1H), 7.26-7.22 (m, 2H), $5.64(\mathrm{~d}, 1 \mathrm{H}), 2.68(\mathrm{~s}, 3 \mathrm{H})$, 2.54 (d, 3H), 1.98 (d, 3H). ${ }^{13} \mathrm{C}-\mathrm{NMR}(101 \mathrm{MHz}, \mathrm{DMSO})$ $\delta 175.13(-\mathrm{C}=\mathrm{O}), 165.85(\mathrm{~N}=\mathrm{C}), 144.15$ (C, Ar), 143.57 (C-S=O), 141.96 (C, Ar), 140.09 (C, Ar), $139.65(\mathrm{~N}-$ $\mathrm{C}=\mathrm{C}), 133.24(\mathrm{CH}, \mathrm{Ar}), 132.11(\mathrm{CH}, \mathrm{Ar}), 131.02(\mathrm{CH}$, Ar), 130.64 (CH, Ar), 129.98 (CH, Ar), $109.45(\mathrm{~S}-\mathrm{C}=\mathrm{C})$, $106.13(\mathrm{~S}-\mathrm{C}=\mathrm{C}), 23.13\left(\mathrm{CH}_{3}\right), 19.20\left(\mathrm{CH}_{3}\right), 17.09\left(\mathrm{CH}_{3}\right)$. (E)-5-((E)-1-(4-Methyl-3-propylthiazol-2(3H)-ylidene) propan-2-ylidene)-2-(methylthio)-4-oxo-3-propyl-4, 5-dihydrothiazol-3-ium-4-methylbenzenesulfonate (5c). Brown crystals; Yield: $84.53 \%$; M.p.: $198{ }^{\circ} \mathrm{C} .{ }^{1} \mathrm{H}$ NMR (400 MHz, DMSO) $\delta 8.23-8.14(\mathrm{~m}, 2 \mathrm{H}), 7.80-7.72$ $(\mathrm{m}, 2 \mathrm{H}), 6.98(\mathrm{~d}, 1 \mathrm{H}), 6.23(\mathrm{~s}, 1 \mathrm{H}), 4.28$ and $3.56(2 \times \mathrm{m}$, $2 \times 2 \mathrm{H}), 2.71(\mathrm{~s}, 3 \mathrm{H}), 2.58(\mathrm{~d}, 3 \mathrm{H}), 2.41(\mathrm{~d}, 3 \mathrm{H}), 2.20(\mathrm{~d}$, $3 \mathrm{H}), 1.57-1.43(\mathrm{~m}, 4 \mathrm{H}), 1.11-0.80(2 \times \mathrm{m}, 2 \times 3 \mathrm{H}) .{ }^{13} \mathrm{C}-$ NMR (101 MHz, DMSO) $\delta 171.02(-\mathrm{C}=\mathrm{O}), 168.25$ $(\mathrm{C}=\mathrm{C}-\mathrm{C}), 142.97(\mathrm{C}-\mathrm{S}=\mathrm{O}), 140.28(\mathrm{C}, \mathrm{Ar}), 138.97(\mathrm{~N}-$ $\mathrm{C}=\mathrm{C}), 130.64(\mathrm{CH}, \mathrm{Ar}), 128.94(\mathrm{CH}, \mathrm{Ar}), 116.17(\mathrm{~N}-$ $\mathrm{C}=\mathrm{C}), 105.82(\mathrm{~S}-\mathrm{C}=\mathrm{C}), 97.58(-\mathrm{C}=\mathrm{C}), 50.18\left(\mathrm{~N}-\mathrm{CH}_{2}\right)$, $46.32\left(\mathrm{~N}-\mathrm{CH}_{2}\right), 24.09\left(\mathrm{CH}_{2}\right), 22.76\left(\mathrm{CH}_{3}\right), 21.98\left(\mathrm{CH}_{3}\right)$, $21.02\left(\mathrm{CH}_{2}\right), 16.95\left(\mathrm{CH}_{3}\right), 15.46\left(\mathrm{CH}_{3}\right), 12.41\left(\mathrm{CH}_{3}\right)$, $11.29\left(\mathrm{CH}_{3}\right)$.

\section{General procedure for the synthesis of thiazolidenium chloride salts (6a-b) and alkylidenthiazolidenium chloride salt (6c)}

At $0{ }^{\circ} \mathrm{C}$, a mixture of $10 \mathrm{~mL}$ of acetonitrile $\left(\mathrm{CH}_{3} \mathrm{CN}\right), 2 \mathrm{mmol}$ of merocyanine or merocarbocyanines already prepared, $0.5 \mathrm{~mL}$ of $12 \mathrm{~N}$ hydrochloric acid $(\mathrm{HCl})$, and $0.8 \mathrm{~mL}$ of hydrogen peroxide $\left(\mathrm{H}_{2} \mathrm{O}_{2}\right) 30 \%$ were placed in a flask under stirring. The reaction can be exothermic. Stirring is maintained for $40 \mathrm{~min}$ or more until a homogeneous mixture appears, then $0.5 \mathrm{~g}$ of Barium chloride $\mathrm{BaCl}_{2}(2$ mmol) contained in $5 \mathrm{~mL}$ of $\mathrm{H}_{2} \mathrm{O}$ was added. The mixture is stirred for 1 to $2 \mathrm{~h}$ until $\mathrm{BaSO}_{4}$ precipitates. 
The mixture was filtered to remove $\mathrm{BaSO}_{4}$, and the thiazolidenium salt was crystallized in methanol $(\mathrm{MeOH})$.

(E)-3,3'-Dibenzyl-4-methyl-4'-oxo-3H,4'H-[2,5'-bithia zolylidene]-3'-ium-chloride (6a). Beige crystals; Yield: 68.20\%; M.p.: $221{ }^{\circ} \mathrm{C} .{ }^{1} \mathrm{H}-\mathrm{NMR}$ (400 MHz, DMSO) $\delta$ 7.68-7.63 (m, 1H), 7.54-7.46 (m, 1H), 7.43-7.37 (m, 1H), 7.34-7.30 (m, 2H), $7.28(\mathrm{~m}, 1 \mathrm{H}), 7.21-7.19(\mathrm{~m}, 2 \mathrm{H}), 6.25$ (d, 1H), 5.20 (d, 1H), 2.19 (d, 3H). ${ }^{13} \mathrm{C}-\mathrm{NMR}(101 \mathrm{MHz}$, DMSO) $\delta 170.11(-\mathrm{C}=\mathrm{O}), 158.64(-\mathrm{N}-\mathrm{C}=\mathrm{C}), 138.86(\mathrm{C}$, $\mathrm{Ar}), 136.57$ (C, Ar), $132.74(\mathrm{CH}, \mathrm{Ar}), 128.69$ (CH, Ar), $128.43(\mathrm{CH}, \mathrm{Ar}), 128.26(\mathrm{CH}, \mathrm{Ar}), 128.13(\mathrm{CH}, \mathrm{Ar})$, 127.78 (CH, Ar), $116.85(\mathrm{~N}-\mathrm{C}=\mathrm{C}), 104.16(\mathrm{~S}-\mathrm{C}=\mathrm{C}), 89.44$ $(-\mathrm{C}=\mathrm{C}-\mathrm{S}-), 51.36\left(\mathrm{~N}-\mathrm{CH}_{2}-\mathrm{C}=\mathrm{C}\right), 48.52\left(\mathrm{~N}-\mathrm{CH}_{2}-\mathrm{C}=\mathrm{C}\right)$, $14.07\left(\mathrm{CH}_{3}\right)$.

(E)-4-Methyl-4'-oxo-3,3'-diphenyl-3H,4'H-[2,5'-bithia zolylidene]-3'-ium (6b). Beige crystals; Yield: 75.92\%; M.p.: $240{ }^{\circ} \mathrm{C} .{ }^{1} \mathrm{H}-\mathrm{NMR}(400 \mathrm{MHz}, \mathrm{DMSO}) \delta 10.79-10.72$ (m, $1 \mathrm{H}), 8.32-8.22(\mathrm{~m}, 2 \mathrm{H}), 7.92-7.80(\mathrm{~m}, 1 \mathrm{H}), 7.52-7.47$ (m, 2H), 7.40-7.35 (m, 1H), 7.28-7.26 (m, 2H), 5.86-5.70 (d, 1H), 2.07 (d, 3H). ${ }^{13} \mathrm{C}-\mathrm{NMR}$ (101 MHz, DMSO) $\delta$ $170.36(-\mathrm{C}=\mathrm{O}), 162.05(\mathrm{~N}-\mathrm{C}=\mathrm{C}), 143.98$ (C, Ar), 141.02 (C, Ar), 138,87 (N-C=C) $132.11(\mathrm{CH}, \mathrm{Ar}), 132.11(\mathrm{CH}$, Ar), 131.02 (CH, Ar), $130.64(\mathrm{CH}, \mathrm{Ar}), 129.98(\mathrm{CH}, \mathrm{Ar})$, $128.62(\mathrm{CH}, \mathrm{Ar}), 108.69(\mathrm{~S}-\mathrm{C}=\mathrm{C}), 105.20(\mathrm{~S}-\mathrm{C}=\mathrm{C}), 18.97$ $\left(\mathrm{CH}_{3}\right)$.

(E)-5-((Z)-1-(5-Methyl-1-propyl-1,3-dihydro-2H-pyrrol2-ylidene)propan-2-ylidene)-4-oxo-3-propyl-4,5-dihy drothiazol-3-ium-chloride (6c). Beige crystals; Yield: 85.22\%; M.p.: $175^{\circ} \mathrm{C} .{ }^{1} \mathrm{H}-\mathrm{NMR}(400 \mathrm{MHz}, \mathrm{DMSO}) \delta 7.15$ $(\mathrm{d}, 1 \mathrm{H}), 6.88(\mathrm{~s}, 1 \mathrm{H}), 4.57-4.51(\mathrm{~m}, 2 \mathrm{H}), 3.95-4.07(\mathrm{~m}$, $2 \mathrm{H}), 2.45(\mathrm{~d}, 1 \mathrm{H}), 2.24(\mathrm{~d}, 1 \mathrm{H}), 1.60-1.47(\mathrm{~m}, 4 \mathrm{H}), 1.12-$ $0.92(\mathrm{~m}, 6 \mathrm{H}) .{ }^{13} \mathrm{C}-\mathrm{NMR}(101 \mathrm{MHz}, \mathrm{DMSO}) \delta 170.53(-$ $\mathrm{C}=\mathrm{O}), 167.46(\mathrm{C}=\mathrm{C}-\mathrm{C}), 139.22(\mathrm{~N}-\mathrm{C}=\mathrm{C}), 116.05(\mathrm{~N}-$ $\mathrm{C}=\mathrm{C}), 105.21 \quad(\mathrm{~S}-\mathrm{C}=\mathrm{C}), 96.38(-\mathrm{C}=\mathrm{C}), 50.12\left(\mathrm{~N}-\mathrm{CH}_{2}\right)$, $49.73\left(\mathrm{~N}-\mathrm{CH}_{2}\right), 23.72\left(\mathrm{CH}_{2}\right), 21.15\left(\mathrm{CH}_{3}\right), 20.69\left(\mathrm{CH}_{2}\right)$, $15.18\left(\mathrm{CH}_{3}\right), 12.05\left(\mathrm{CH}_{3}\right), 10.91\left(\mathrm{CH}_{3}\right)$.

\section{General procedure for the study of the transformation of merocyanines and merocarbocyanines salts to TTTAFs}

The thiazolidenium sulfonate salts $(7 \mathbf{a}-\mathbf{c})(0.05 \mathrm{~mol})$ or thiazolidenium chloride salts $(\mathbf{8 a}-\mathbf{c})(0.05 \mathrm{~mol})$ were introduced into the electrolysis cell containing $0.1 \mathrm{M}$ solution of tetra-n-butylammonium bromide $\mathrm{TBAB}$
$(\mathrm{NBu} 4 \mathrm{Br}), 5 \mu \mathrm{L}$ of perchloric acid and, $50 \mathrm{~mL}$ of acetonitrile. The environment was degassed by a stream of nitrogen for $5 \mathrm{~min}$ and maintained under an inert atmosphere. The reduction of salt is carried out on a platinum electrode. The voltammograms were recorded at ambient temperature with a scanning speed of 100 $\mathrm{mV}^{-1} \mathrm{~S}^{-1}$. The selected scanning range was -2000 to $+2000 \mathrm{mV}$ and 0 to $2000 \mathrm{mV}$.

\section{- RESULTS AND DISCUSSION}

\section{Chemical Synthesis Part}

In this study, we described practical approaches for the preparation of thiazolidenium salts obtained from merocyanine derivatives. Firstly, thioxothiazolidinones (1a-c), alkylidenethioxotiazolidinone (3c), and thiazoline thiones (2a-c), as well as the thiazolium salts (3a-b), were prepared. The coupling of such compounds results in the formation of thiazolydenethioxothiazolidinones (4ab) and alkylidenthiazolidenethioxothiazolidinone (4c), which are subsequently converted to thiazolidenium sulfonate salts (5a-b), thiazolidenium chloride salts (6ab), alkylidenthiazolidenium sulfonate salt $(\mathbf{5 c})$, and alkylidenthiazolidenium chloride salt (6c) successively. The synthetic strategies for the preparation of these salts are grouped in Scheme 1 and Scheme 2. Concerning the first scheme, thioxothiazolidinones (1a-b) were synthesized by a cyclo condensation of chloroacetic acid with ammonium dithiocarbamate salt (DTC) [37]. DTCs were obtained from the following primary amines: $\mathrm{BnNH}_{2}, \mathrm{PhNH}_{2}$, and $n-\mathrm{PrNH}_{2}$ that ultimately with $\mathrm{CH}_{3} \mathrm{COOH}$ leads to the formation of the corresponding products 1a (Beige crystals; 68\%), and 1b (Yellow Crystals; 59\%) successively. Thiazolinethiones 2a (Beige crystals; $83.55 \%$ ) and $\mathbf{2 b}$ (Beige crystals; 61.55\%) were obtained by the reaction between chloroacetone and the same preceding dithiocarbamate salts (DTCs). The treatment of thiazolinethiones with iodomethane in the presence of acetone leads to the formation of the thiazolium salt 3a (White crystals; 60.58\%) and 3b (Beige crystals; 53.64\%). Finally, merocyanines 4a (Yellow crystals; 84.21\%) and 4b (Yellow crystals; 81.53\%) were obtained using two methods: (i) The first

Manel Khiat et al. 


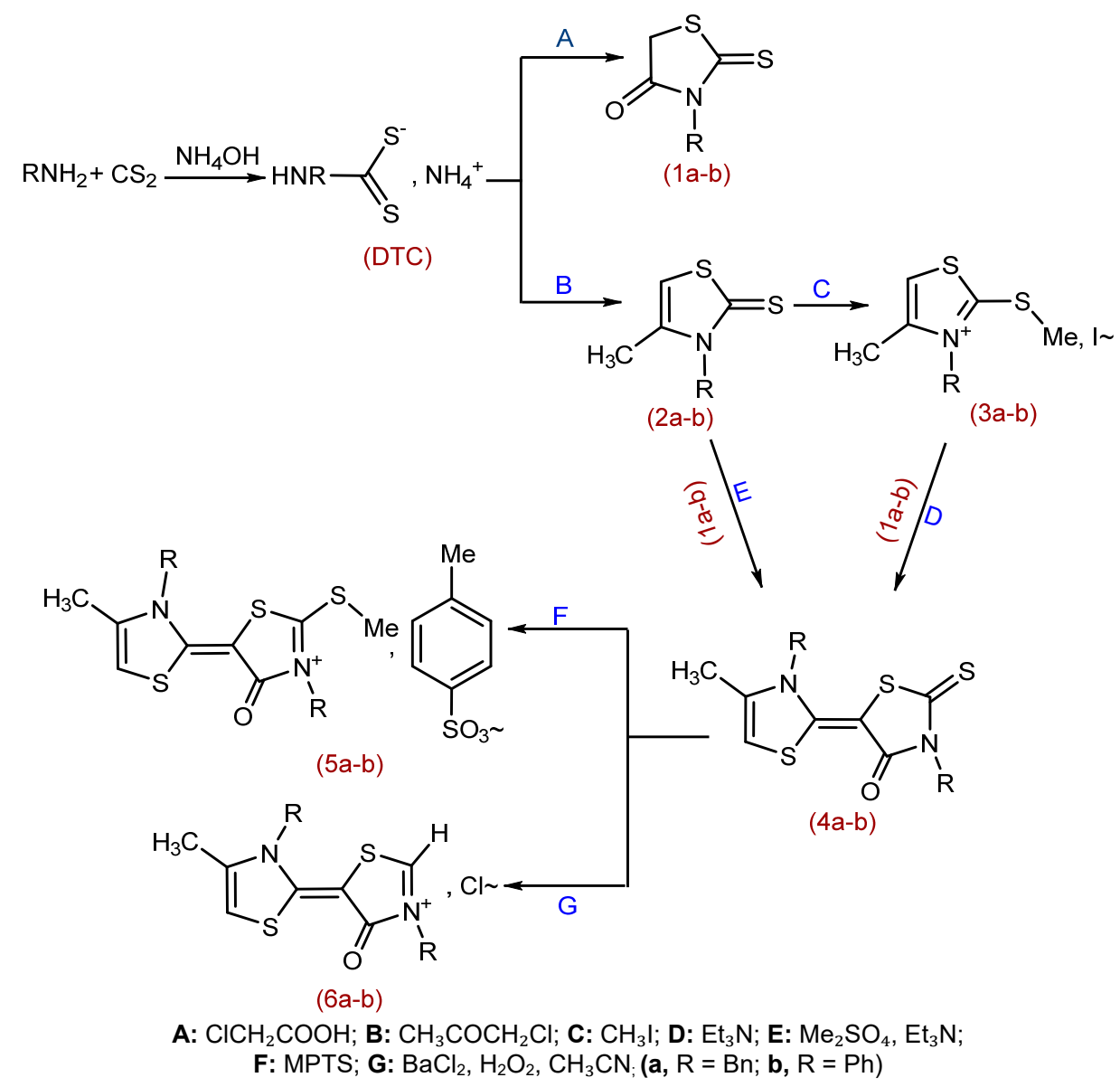

Scheme 1. The synthetic procedure of thiazolidenium sulfonate salts $(\mathbf{5 a}-\mathbf{b})$ and thiazolidenium chloride salts $(\mathbf{6 a}-\mathbf{b})$

was a coupling between a neutral heterocycle (thioxothiazolidinone 1a-b) and a cationic heterocycle (thiazolium salts 3a-b) in the presence of triethylamine. (ii) The second method was an in situ reaction between compounds (2a-b) and compounds (1a-b) in the presence of dimethyl sulfate and triethylamine.

Comparing the two methods, the second one is easier and faster than the first one. The reaction of the prepared merocyanines (4a-b) with methyl $p$-toluene sulfonate (MPTS) in the presence of acetone leads to the formation of the thiazolideniumsulfonate salts $\mathbf{5 a}$ (Brown crystals; 78.96\%) and 5b (Brown crystals; 71.24\%). The reaction of the same prepared merocyanines $(4 \mathbf{a}-\mathbf{b})$ with barium chloride $\left(\mathrm{BaCl}_{2}\right)$ in the presence of acetonitrile and hydrogen peroxide leads to the formation of the thiazolidenium chloride salts 6a (Beige crystals; 68.20\%), and $\mathbf{6 b}$ (Beige crystals; 75.92\%). Another strategy was followed for the preparation of alkylidenthiazolidene- thioxothiazolidinone (merocarbocyanines) 4c (Purple crystals; $87.32 \%$ ) and their salts $5 \mathrm{c}$ (Brown crystals; $84.53 \%$ ), and 6c (Beige crystals; 85.22\%), which is illustrated in Scheme 2. Thioxothiazolidinones 1c (Brown oily liquid; 62\%) was obtained by the same previous method. The reaction between compound 1c (containing active methylene in position 5) with acetone (containing a carbonyl group) in the presence of ammonium hydroxide used as catalyst, whit heating under reflux for $20 \mathrm{~h}$, leads to the formation of alkylidenethioxo tiazolidinone 3c (Orange crystals; 59\%), according to the knoevenagel condensation [38]. Thiazolinethione 2c (Beige crystals; 76.52\%) was obtained by the same previous method. The compound 4c (Purple crystals; 87.32\%) was obtained by an in situ reaction between a compound $3 c$ and $2 c$ in the presence of dimethyl sulfate and triethylamine. The alkyliden thiazolideniumsulfonate salt $\mathbf{5 c}$ (Brown crystals; 84.53\%) 


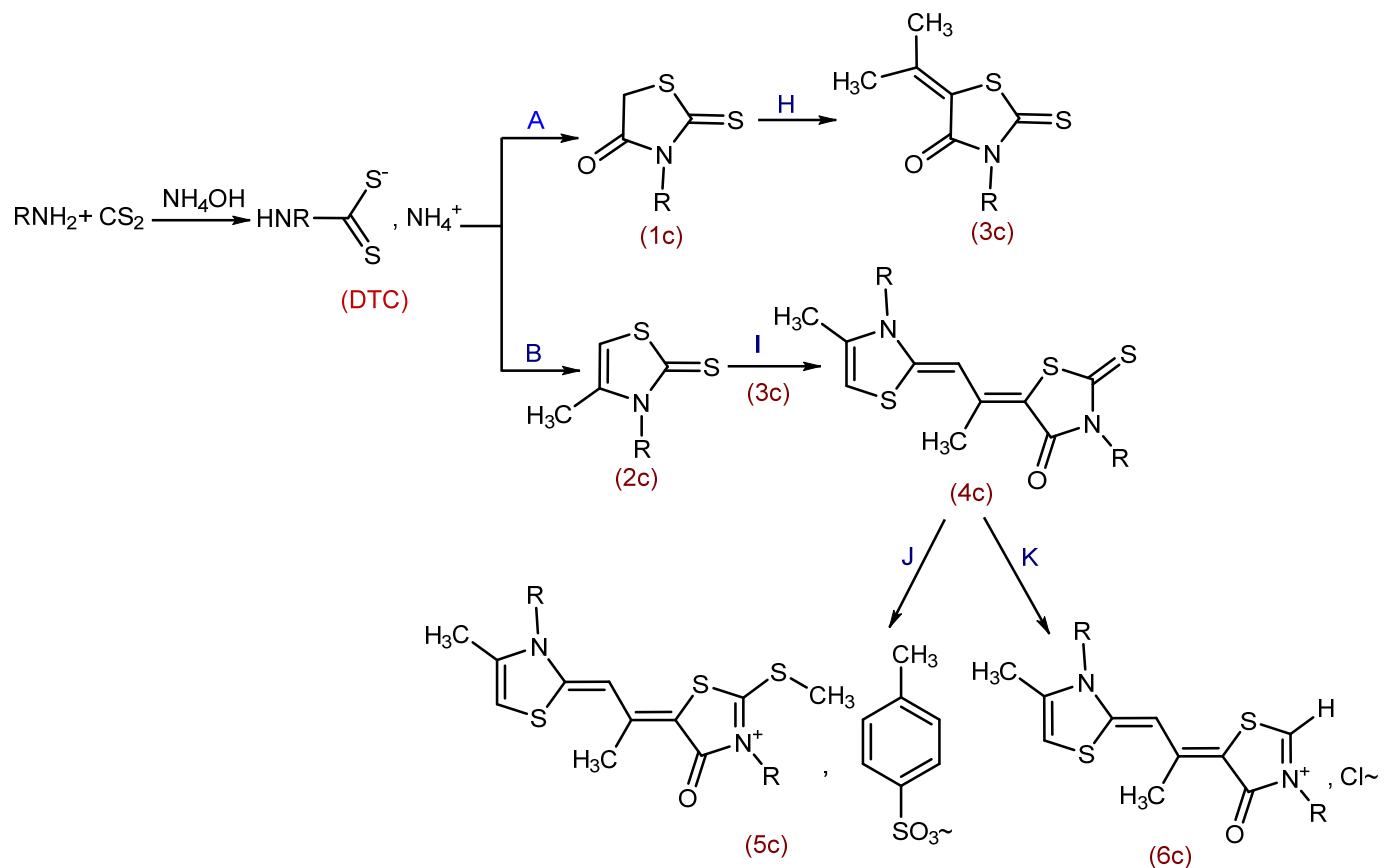

A: $\mathrm{ClCH}_{2} \mathrm{COOH}$; B: $\mathrm{CH}_{3} \mathrm{COCH}_{2} \mathrm{Cl} ; \mathrm{H}: \mathrm{CH}_{3}(\mathrm{CO}) \mathrm{CH}_{3}$; I: $\mathrm{Me}_{2} \mathrm{SO}_{4}, \mathrm{Et}_{3} \mathrm{~N}$; J: MPTS, DMF; K: $\mathrm{BaCl}_{2}, \mathrm{H}_{2} \mathrm{O}_{2}, \mathrm{CH}_{3} \mathrm{CN} ;(\mathbf{c}, \mathrm{R}=\mathrm{Pr})$

Scheme 2. The synthetic procedure of alkylidenthiazolidenium sulfonate salt (5c) and alkylidenthiazolidenium chloride salt $(\mathbf{6 c})$

was obtained by the reaction of compound $4 \mathrm{c}$ with MPTS in the presence of DMF and acetone. The treatment of the compound $\mathbf{4 c}$ with a mixture of hydrogen peroxide $\left(\mathrm{H}_{2} \mathrm{O}_{2}\right)$ and barium chloride $\left(\mathrm{BaCl}_{2}\right)$ in the presence of acetonitrile leads to the formation of alkylidenthiazolidenium chloride salt $\mathbf{6 c}$ (Beige crystals; $85.22 \%$ ) with precipitation of $\mathrm{BaSO} 4$, which was removed by filtration through celite, The method used to generate the chloride salt $\mathbf{6 c}$ was inspired from the work of Lorcy and Guérin [39] and Yano et al. [40]. The yields of the synthesized compounds are satisfactory (60-87\%), and the data obtained from IR, NMR, MS spectroscopic analyses were in good agreement with the proposed structures of the synthesized molecules.

\section{Electrochemical (Cyclic-Voltammetry) Study Part}

By studying the behavior of thiazolidenium sulfonate salts (5a-b), thiazolidenium chloride salts (6ab), alkylidenthiazolidenium sulfonate salt $(5 \mathbf{c})$, and alkylidenthiazolidenium chloride salt $(\mathbf{6 c})$ in a voltammetric cell on a platinum electrode; we noticed that they turn into TTTAFs. Because of the conjugation existing in the structure of these salts, there is delocalization of the $\pi$-electrons in their molecules, which have the effect of generating a radical. The latter is dimerized into an intermediate (each salt gives an intermediate: I, II, III, IV). The reduction of each intermediate derivative, after elimination of two $\mathrm{MeS}^{-}$or $\mathrm{H}^{-}$groups, to the formation of TTTAFs (7a-b, 7'a-b, 8c, and $\mathbf{8}^{\prime} \mathbf{c}$ ) successively, according to Scheme 3, 4 (see SI). It should be noted that the presence of acid facilitates the elimination of the thiomethyl group and facilitates the formation of TTTAF.

\section{Interpretation of Voltammograms}

The range of the cyclic scan chosen was (-2000 to $+2000 \mathrm{mV})$ and $(0$ to $+2000 \mathrm{mV})$ from right to left with a constant speed of $100 \mathrm{mV} / \mathrm{s}$ for all voltammograms.

\section{Solvent and support electrolyte voltammograms}

From the two scans below, it is noted that there are no oxidation or reduction peaks. Therefore, there has been no electrochemical phenomenon on the solvent or the electrolyte support, despite the appearance of weak "bumps" probably due to traces of oxygen (see Fig. 2). 


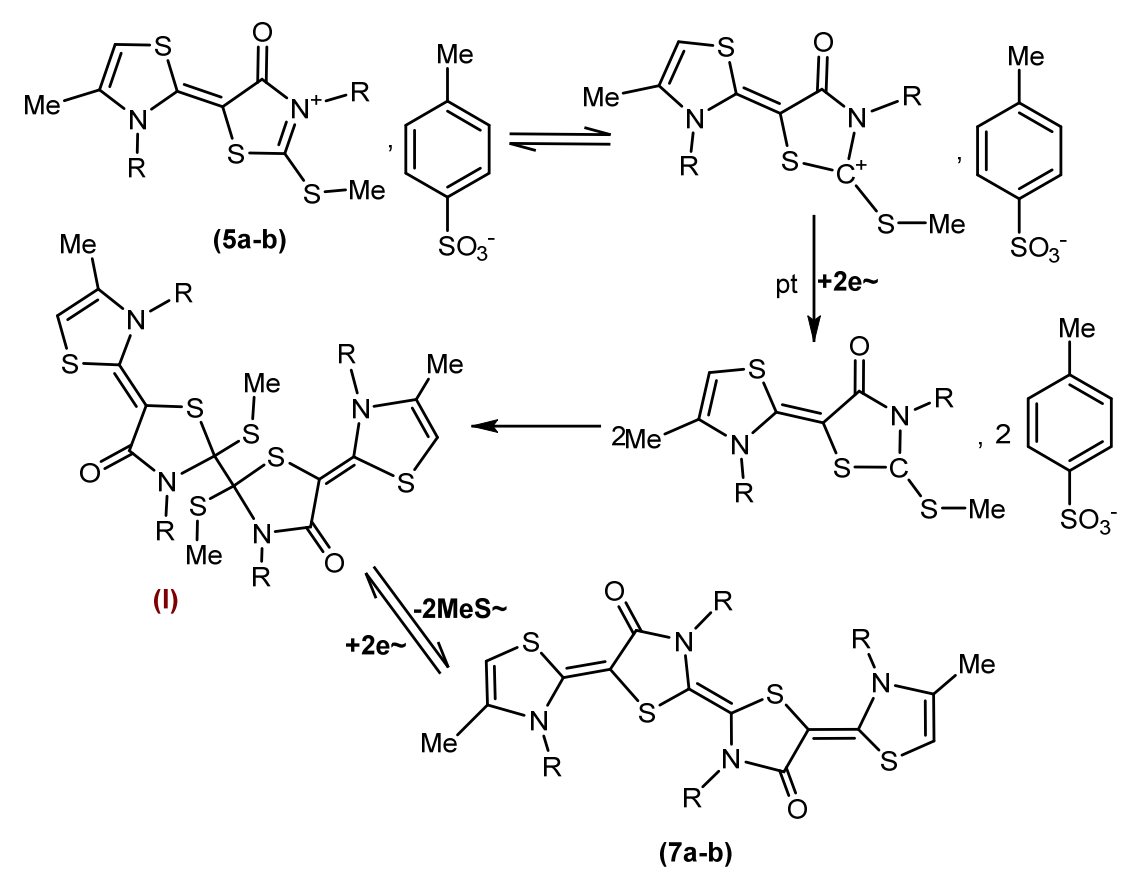

Scheme 3. The strategy of electrochemical transformation of thiazolidenium sulfonate salts (5a-b) into TTTAFs (7a-b)<smiles>[R]N1C(C)=CSC1=C1C=[N+]([R])C(=O)S1</smiles><smiles></smiles>

$6(a-b)$

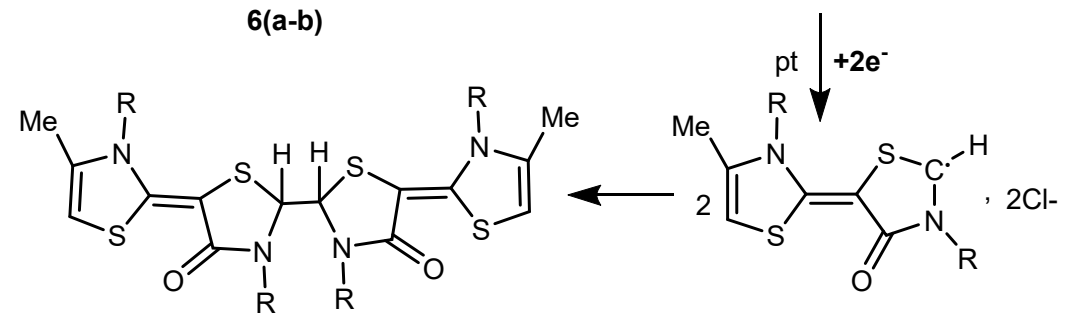

(II)<smiles></smiles><smiles></smiles>

Scheme 4. The strategy of electrochemical transformation of thiazolideniumchloride salts (6a-b) into TTTAFs (7'a-b)

Voltammograms of the merocyanines $4 a, 4 b$, and merocarbocyanine 4c (Fig. 3, and SI)

According to the appearance of the voltammograms of the three compounds $4 \mathbf{a}, \mathbf{4 b}, \mathbf{4 c}$, two peaks of oxidation are observed in each scan at different potentials; (compound 4a: $\left.\mathrm{Ep}_{\mathrm{a} 1}=1.15 ; \mathrm{Ep}_{\mathrm{a} 2}=1.65\right)$, (compound 4b:
$\mathrm{Ep}_{\mathrm{a} 1}=1.15 ; \mathrm{Ep}_{\mathrm{a} 2}=1.65$ ), and (compound 4c: $\mathrm{Ep}_{\mathrm{a} 1}=1.15 ;$ $\left.\mathrm{Ep}_{\mathrm{a} 2}=1.6\right)$. A single reduction peak for each scan are also observed at different potentials; (compound 4a: $\mathrm{Ep}_{\mathrm{c} 2}=$ 0.63, compound $4 \mathrm{~b}$ : $\mathrm{Ep}_{\mathrm{c} 2}=0.63$, and compound $4 \mathrm{c}$ : $\mathrm{Ep}_{\mathrm{c} 2}$ $=0.63$ ).

According to the peaks, the first wave of oxidation 

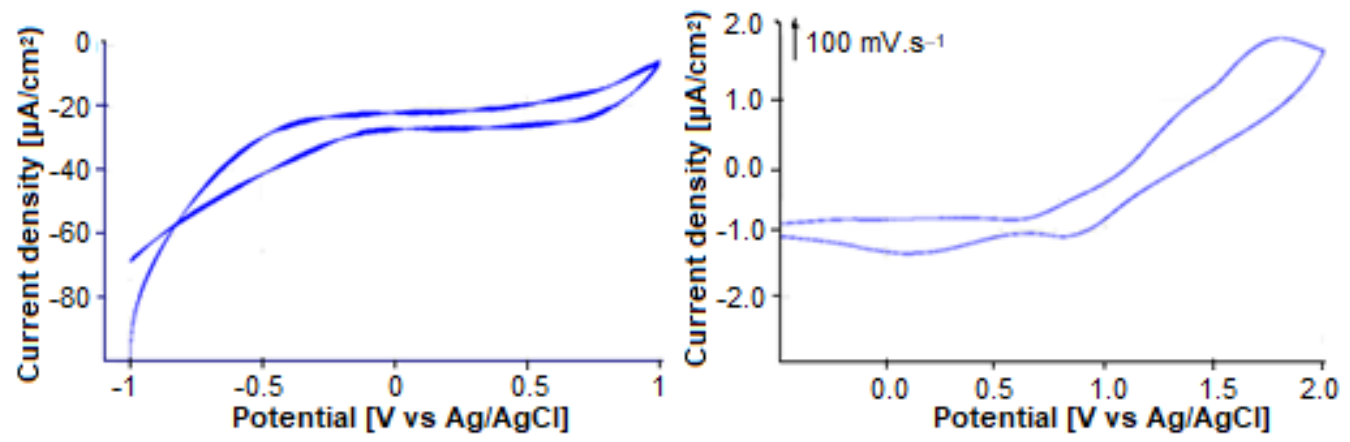

$\left(\mathrm{CH}_{3} \mathrm{CH}, \mathrm{HClO}_{4}, \mathrm{Pt}, \mathrm{Ag} / \mathrm{AgCl}, 0.1 \mathrm{~V} / \mathrm{s}\right)$

(TBAB, $\mathrm{CH}_{3} \mathrm{CN}, \mathrm{NBu}_{4} \mathrm{Br}, \mathrm{HClO}_{4}, \mathrm{Pt}, \mathrm{Ag} / \mathrm{AgCl}, 0.1 \mathrm{~V} / \mathrm{s}$ )

Fig 2. Solvent and support electrolyte voltammograms
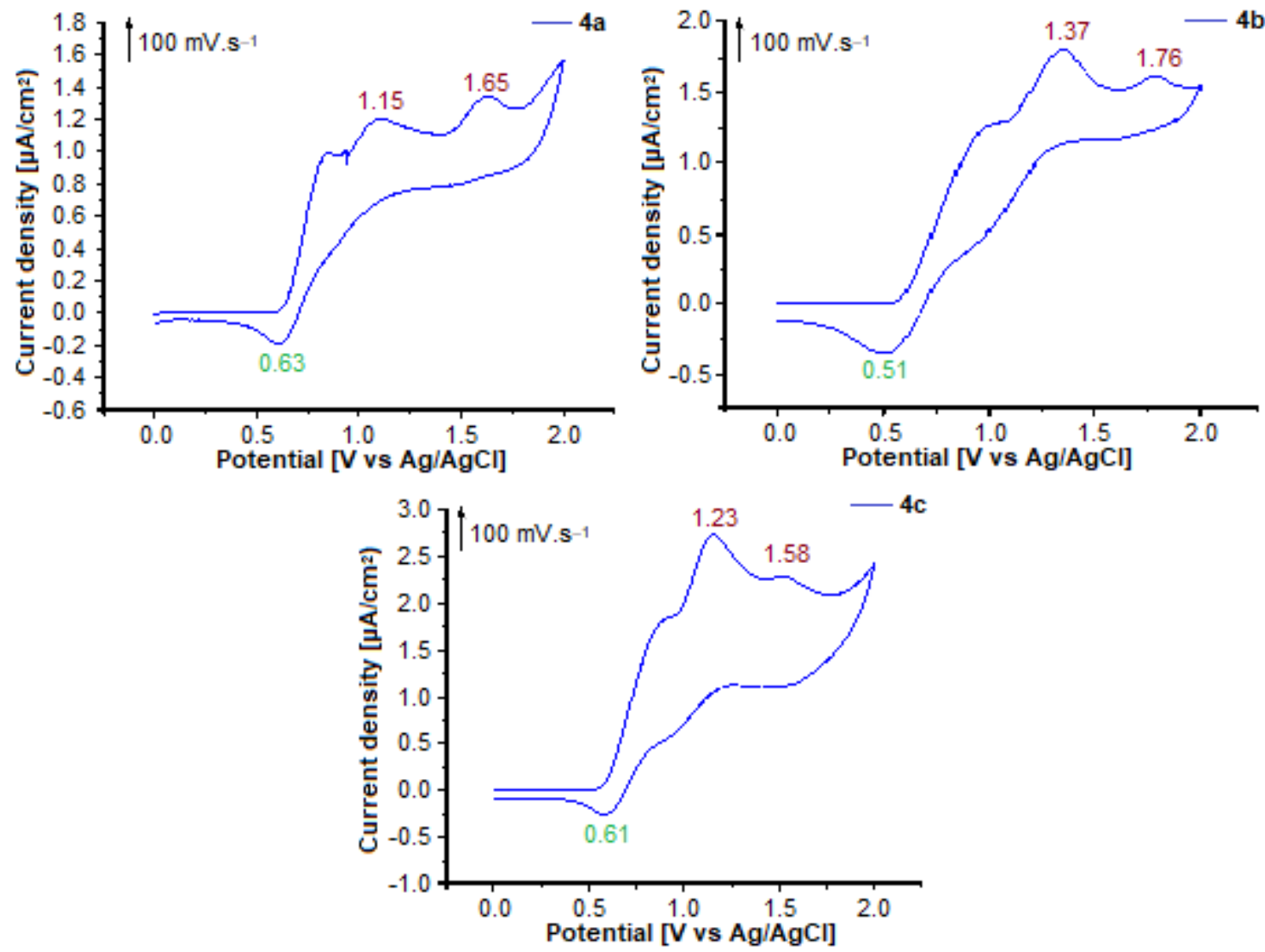

Fig 3. Voltammograms of compounds $4 \mathbf{a}, 4 \mathbf{b}, 4 \mathbf{c}\left(\mathrm{CH}_{3} \mathrm{CN}, \mathrm{NBu}_{4} \mathrm{Br} 0.1 \mathrm{M}, \mathrm{HClO}_{4}\right.$, pt, $\left.\mathrm{Ag} / \mathrm{AgCl}, 0.1 \mathrm{~V} / \mathrm{s}\right)$

is reversible, but not the second (irreversible). This indicates that there was the oxidation of two electrons and the reduction of only one electron for each compound. Therefore, it is an irreversible anodic oxidation process, and consequently, there is no coupling phenomenon on these precursors.

Note: $4 \mathbf{a}, \mathbf{4 b}$, and $\mathbf{4 c}$ are the precursors of the thiazolidenium salts: (4a precursor of $5 \mathbf{a}$ and $6 \mathbf{6}),(4 \mathbf{b}$ precursor of $5 b$ and $6 b)$, (4c precursor of $5 c$ and $6 c)$.

\section{Merocyanines salts voltammograms}

Voltammograms of thiazolidenium sulfonate salts 5a, 5b (Fig. 4, and $\mathrm{SI}$ ), and thiazolidenium chloride salts $\mathbf{6 a}, \mathbf{6 b}$ (Fig. 5, and SI). The four voltammograms indicate a well-defined redox process, which corresponds to the reversible oxidations of the four merocyanine salts $\mathbf{5 a}, \mathbf{5 b}, \mathbf{6 a}, \mathbf{6 b}$. The anodic peaks (oxidation) of each compound are observed at $\left(5 \mathrm{a}: \mathrm{Ep}_{\mathrm{a} 1}=1.24 ; \mathrm{Ep}_{\mathrm{a} 2}=1.60\right)$, $\left(5 \mathbf{b}: \mathrm{Ep}_{\mathrm{a} 1}=0.95 ; \mathrm{Ep}_{\mathrm{a} 2}=1.30\right),\left(6 \mathrm{a}: \mathrm{Ep}_{\mathrm{a} 1}=0.93 ; \mathrm{Ep}_{\mathrm{a} 2}=1.90\right)$, 

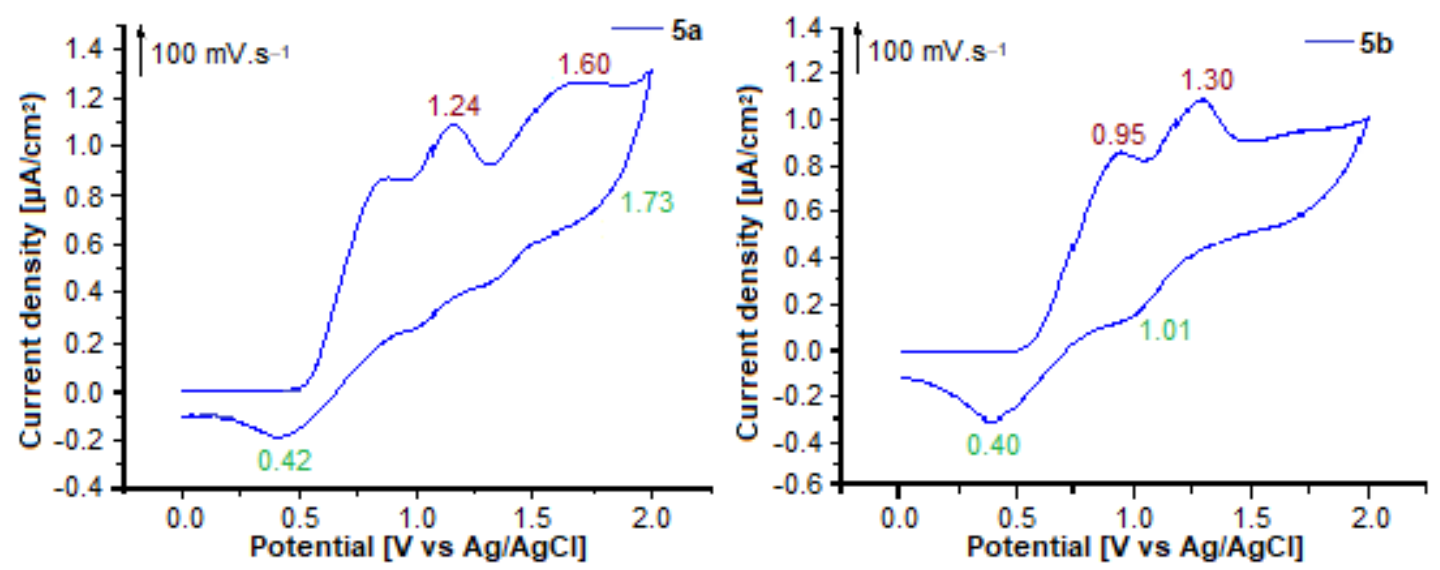

Fig 4. Voltammograms of compounds $\mathbf{5 a}, \mathbf{5 b}\left(\mathrm{CH}_{3} \mathrm{CN}, \mathrm{NBu}_{4} \mathrm{Br} 0.1 \mathrm{M}, \mathrm{HClO}_{4}, \mathrm{pt}, \mathrm{Ag} / \mathrm{AgCl}, 0.1 \mathrm{~V} / \mathrm{s}\right)$
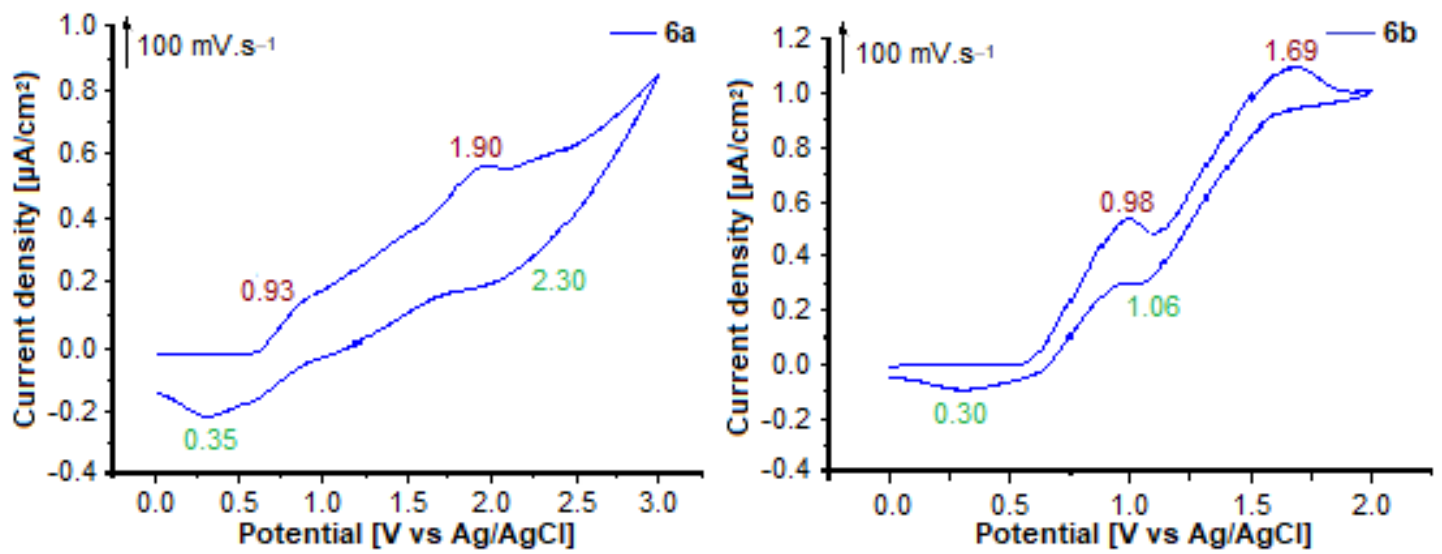

Fig 5. Voltammograms of compounds $\mathbf{6 a}, \mathbf{6 b}\left(\mathrm{CH}_{3} \mathrm{CN}, \mathrm{NBu}_{4} \mathrm{Br} 0.1 \mathrm{M}, \mathrm{HClO}_{4}, \mathrm{pt}, \mathrm{Ag} / \mathrm{AgCl}, 0.1 \mathrm{~V} / \mathrm{s}\right)$

(6b: $\left.\mathrm{Ep}_{\mathrm{a} 1}=0.98 ; \mathrm{Ep}_{\mathrm{a} 2}=1.69\right)$. The cathodic peaks (reduction) of each compound are observed at $\left(\mathbf{5 a}: \mathrm{Ep}_{\mathrm{c} 1}=\right.$ $\left.1.73, \mathrm{Ep}_{\mathrm{c} 2}=0.42\right),\left(5 \mathbf{b}: \mathrm{Ep}_{\mathrm{c} 1}=1.01, \mathrm{Ep}_{\mathrm{c} 2}=0.40\right),\left(\mathbf{6 a}: \mathrm{Ep}_{\mathrm{c} 1}=\right.$ 2.30, $\left.\mathrm{Ep}_{\mathrm{c} 2}=0.35\right),\left(6 \mathbf{b}: \mathrm{Ep}_{\mathrm{c} 1}=1.06, \mathrm{Ep}_{\mathrm{c} 2}=0.30\right)$.

According to the scans and peaks, the two waves of oxidation are reversible in each voltammogram. This indicates that there was a transfer of electrons: the oxidation of two electrons and the reduction of two electrons for each compound. The redox of the first two electrons corresponds to the generation of the radical, which is subsequently dimerized to an intermediate, and the redox of the other two electrons corresponds to the transformation of the latter into TTTAFs. Therefore the thiazolidenium sulfonate salts $\mathbf{5 a}, \mathbf{5 b}$ have been transformed into TTTAFs $\mathbf{7 a}, \mathbf{b}$ and that the thiazolidenium chloride salts $\mathbf{6 a}, \mathbf{b}$ have been transformed into TTTAFs 7’a,b.

Merocarbocyanines salts voltammograms Voltammograms of alkylidenthiazolideniumsulfo nate salt $5 \mathrm{c}$ and alkylidenthiazolidenium chloride salt 6c (Fig. 6, and SI). The scans of the two voltammograms allow us to observe two reversible waves with two anode peaks and two cathodic responses. The two peaks corresponding to the oxidation of the salts are located at $\left(\mathbf{5 c}: \mathrm{Ep}_{\mathrm{a} 1}=0.95 ; \mathrm{Ep}_{\mathrm{a} 2}=1.31\right),\left(\mathbf{6 c}: \mathrm{Ep}_{\mathrm{a} 1}\right.$ $\left.=0.80 ; \mathrm{Ep}_{\mathrm{a} 2}=1.39\right)$, and the other two peaks corresponding to the reduction of the salts are located at $\left(5 \mathrm{c}: \mathrm{Ep}_{\mathrm{c} 1}=1.56, \mathrm{Ep}_{\mathrm{c} 2}=0.39\right),\left(6 \mathrm{c}: \mathrm{Ep}_{\mathrm{c} 1}=1.60, \mathrm{Ep}_{\mathrm{c} 2}=0.71\right)$, this indicates that there was a transfer of electrons: oxidation and reduction of two electrons for each compound and therefore the transformation of salts $\mathbf{5 c}$ and $\mathbf{6 c}$ at TTTAF $\mathbf{8 c}$ and $\mathbf{8}^{\prime} \mathbf{c}$, respectively. 

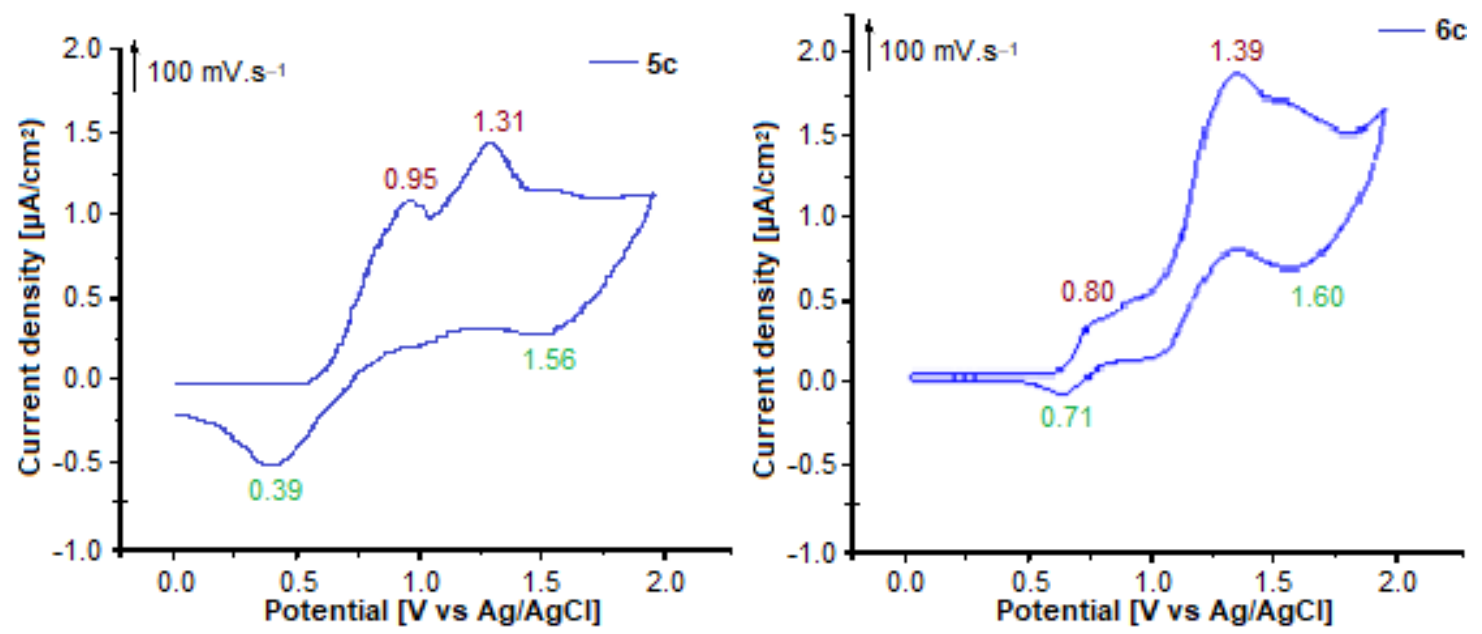

Fig 6. Voltammograms of compounds $5 \mathbf{c}, \mathbf{6 c}\left(\mathrm{CH}_{3} \mathrm{CN}, \mathrm{NBu}_{4} \mathrm{Br} 0.1 \mathrm{M}, \mathrm{HClO}_{4}, \mathrm{pt}, \mathrm{Ag} / \mathrm{AgCl}, 0.1 \mathrm{~V} / \mathrm{s}\right)$

Summary Table of the Different OxidationReduction Potentials of the ThiazolideniumSulfonate and Chloride Salts and Their Precursors

We present, in Table 1, 2, 3 a summary of the different oxidation-reduction potentials of each merocyanines and merocarbocyanines salts $\mathbf{5 a}, \mathbf{6 a}, \mathbf{5 b}, \mathbf{6} \mathbf{b}$, $\mathbf{5 c}$, $\mathbf{6 c}$ with their precursor's merocyanines and merocarbocyanines $\mathbf{4 a}, \mathbf{4 b}, \mathbf{4 c}$ successively, to compare the results.

\section{Confirmation}

The cyclic Voltammetry method allowed us to follow the behavior of merocyanine salts (thiazolideniumsulfonate salts $\mathbf{5 a}, \mathbf{5 b}$, and thiazolidenium chloride salts $\mathbf{6 a}, \mathbf{6 b}$, and merocarbocyanine salts thiazolideniumsulfonate salts $\mathbf{5 c}$ and thiazolidenium chloride salts $\mathbf{6 c}$ at different potentials. By comparing the voltammograms and the different potentials of the salts with their precursors $\mathbf{4 a}, \mathbf{4 b}, \mathbf{4 c}$ taken as references, we observe that for the salts, we have a redox system of 4 $\pi$-electrons and consequently their delocalization, which leads to the coupling of these latest; It is, therefore, easy to confirm their transformation to TTTAFs $\mathbf{7 a}, \mathbf{7 b}, \mathbf{7} \mathbf{a}^{\prime}$, $\mathbf{7 b}, \mathbf{8 c}, \mathbf{8 c}$ ' as shown in Scheme 3, 4 (see SI). For the precursor molecules, there was not a redox system, and therefore no coupling.

The confirmation of the presence of TTTAFs was made only by an in situ electrochemical detection method

Table 1. Differents oxidation-reduction potentials of the compounds (4a, 5a, 6a)

\begin{tabular}{|c|c|c|c|c|c|c|c|c|}
\hline \multicolumn{3}{|c|}{$\begin{array}{c}\text { Potentials of the compound } \\
\text { (4a) (volt) }\end{array}$} & \multicolumn{3}{|c|}{$\begin{array}{l}\text { Potentials of the compound } \\
\text { (5a) (volt) }\end{array}$} & \multicolumn{3}{|c|}{$\begin{array}{l}\text { Potentials of the compound } \\
\text { (6a) (volt) }\end{array}$} \\
\hline $\mathrm{Ep}_{\mathrm{a} 1}$ & $\mathrm{Ep}_{\mathrm{a} 2}$ & $\Delta \mathrm{E}_{\text {Pox }}$ & $\mathrm{Ep}_{\mathrm{a} 1}$ & $\mathrm{Ep}_{\mathrm{a} 2}$ & $\Delta \mathrm{E}_{\text {Pox }}$ & $E p_{a 1}$ & $E p_{\mathbf{a} 2}$ & $\Delta \mathrm{E}_{\text {Pox }}$ \\
\hline+1.15 & +1.65 & +0.50 & +1.24 & +1.60 & +0.36 & +0.93 & +1.92 & +0.99 \\
\hline $\mathbf{E p}_{\mathrm{c} 1}$ & $\mathbf{E p}_{\mathrm{c} 2}$ & $\Delta \mathrm{E}_{\text {Red }}$ & $\mathrm{Ep}_{\mathrm{c} 1}$ & $\mathrm{Ep}_{\mathrm{c} 2}$ & $\Delta \mathrm{E}_{\mathrm{Red}}$ & $\mathrm{Ep}_{\mathrm{c} \mathbf{c}}$ & $\mathrm{Ep}_{\mathrm{c} 2}$ & $\Delta \mathrm{E}_{\mathrm{Red}}$ \\
\hline 0 & +0.63 & +0.63 & +0.42 & +1.73 & +1.31 & +0.35 & +2.30 & +1.95 \\
\hline
\end{tabular}

Table 2. Differents oxidation-reduction potentials of the compounds $(\mathbf{4 b}, \mathbf{5 b}, \mathbf{6 b})$

\begin{tabular}{|c|c|c|c|c|c|c|c|c|}
\hline \multicolumn{3}{|c|}{$\begin{array}{l}\text { Potentials of the compound } \\
\text { (4b) (volt) }\end{array}$} & \multicolumn{3}{|c|}{$\begin{array}{l}\text { Potentials of the compound } \\
\qquad(5 b) \text { (volt) }\end{array}$} & \multicolumn{3}{|c|}{$\begin{array}{l}\text { Potentials of the compound } \\
\text { (6b) (volt) }\end{array}$} \\
\hline$E p_{a 1}$ & $\mathrm{Ep}_{\mathbf{a} 2}$ & $\Delta \mathrm{E}_{\text {Pox }}$ & $E p_{a 1}$ & $\mathrm{Ep}_{\mathbf{a} 2}$ & $\Delta \mathrm{E}_{\text {Pox }}$ & $E p_{a 1}$ & $\mathrm{Ep}_{\mathbf{a} 2}$ & $\Delta \mathrm{E}_{\text {Pox }}$ \\
\hline+1.37 & +1.76 & +0.39 & +0.95 & +1.30 & +0.35 & +0.98 & +1.69 & +0.71 \\
\hline $\mathrm{Ep}_{\mathrm{c} 1}$ & $\mathrm{Ep}_{\mathrm{c} 2}$ & $\Delta \mathrm{E}_{\text {Red }}$ & $\mathrm{Ep}_{\mathrm{cl}}$ & $\mathrm{Ep}_{\mathrm{c} 2}$ & $\Delta \mathbf{E}_{\text {Red }}$ & $\mathrm{Ep}_{\mathrm{c} 1}$ & $\mathrm{Ep}_{\mathrm{c} 2}$ & $\Delta \mathrm{E}_{\mathrm{Red}}$ \\
\hline 0 & +0.51 & +0.51 & +0.4 & +1.01 & +0.61 & +0.30 & +1.06 & +0.76 \\
\hline
\end{tabular}


Table 3. Differents oxidation-reduction potentials of the compounds $(4 c, 5 c, 6 c)$

\begin{tabular}{|c|c|c|c|c|c|c|c|c|}
\hline \multicolumn{3}{|c|}{$\begin{array}{l}\text { Potentials of the compound } \\
\qquad(4 c) \text { (volt) }\end{array}$} & \multicolumn{3}{|c|}{$\begin{array}{l}\text { Potentials of the compound } \\
\qquad(5 \mathrm{c}) \text { (volt) }\end{array}$} & \multicolumn{3}{|c|}{$\begin{array}{l}\text { Potentials of the compound } \\
\qquad(6 c) \text { (volt) }\end{array}$} \\
\hline $\mathrm{Ep}_{\mathrm{a} 1}$ & $\mathrm{Ep}_{\mathrm{a} 2}$ & $\Delta \mathrm{E}_{\text {Pox }}$ & $\mathrm{Ep}_{\mathrm{a} 1}$ & $\mathrm{Ep}_{\mathrm{a} 2}$ & $\Delta E_{\text {Pox }}$ & $\mathbf{E p}_{\mathrm{a} 1}$ & $\mathrm{Ep}_{\mathrm{a} 2}$ & $\Delta \mathrm{E}_{\text {Pox }}$ \\
\hline+1.23 & +1.58 & +0.35 & +0.95 & +1.31 & +0.36 & +0.80 & +1.39 & +0.59 \\
\hline $\mathrm{Ep}_{\mathrm{cl}}$ & $\mathrm{Ep}_{\mathrm{c} 2}$ & $\Delta \mathrm{E}_{\mathrm{Red}}$ & $\mathrm{Ep}_{\mathrm{cl}}$ & $\mathbf{E p}_{\mathrm{c} 2}$ & $\Delta \mathbf{E}_{\mathrm{Red}}$ & $\mathrm{Ep}_{\mathrm{cl}}$ & $\mathbf{E p}_{\mathrm{c} 2}$ & $\Delta \mathrm{E}_{\mathrm{Red}}$ \\
\hline 0 & +0.61 & +0.61 & +0.39 & +1.56 & +1.17 & +0.71 & +1.60 & +0.89 \\
\hline
\end{tabular}

under an inert atmosphere and not with usual spectroscopic methods (NMR, MS, UV, etc.) for the reason that the TTTAFs are very unstable species and very reactive to air and therefore their isolation causes their degradation.

According to the literature [40-42], DTDAFs (analogs of TTTAFs), which are also unstable in air and not isolable, have been transformed into DTDAF ${ }^{2+}$ dications more stable and isolable; as a perspective, we can transform the TTTAFs that we could not isolate to TTTAF $^{4+}$ tetracations more stable and isolable, to characterize them by spectroscopic methods.

\section{Structure-Property Relationship of TTTAFs}

TTTAFs are nitrogen analogs of TTFs (family of electroactive donors) where rings thiol have been replaced by rings thiazole (presence of nitrogen which can undergo oxidation or bind to a metal center), which gives them a very high electro-donor character and which make them very reactive to electron-acceptor compounds and therefore they can be used in assembly reactions to obtain new charge transfer complexes having superconducting properties.

TTTAFs have a $\pi$-conjugate system, which gives them a conductive character, and consequently, they have redox properties.

TTTAFs have very high electron donor properties, and therefore are very unstable and reactive in air, to make them react with other molecules such as electronattracting compounds; we must use an electrochemical detection method In situ in a cell, under an inert atmosphere (medium degassed with nitrogen).

\section{- CONCLUSION}

In summary, in this work, we sought to obtain TTTAFs from salts of merocyanines used as precursor molecules. After various attempts, by applying electrosynthesis (cyclic voltammetry) to these salts and by following their behavior in situ in the electrochemical cell, we were able to lead to the intramolecular coupling of these latter and consequently to the formation of TTTAFs, which was confirmed by the displacement of the oxidation-reduction waves and the variation of the observed potentials. From the structure of TTTAFs, which contains electron donor groups (nitrogen and sulfur) and several double bonds, we can consider them as $\pi$-electron donor's molecules, which will be tested subsequently against another $\pi$-electron acceptor structure.

\section{- SUPPORTING INFORMATION (SI)}

Copies of the original spectra (IR, ${ }^{1} \mathrm{H}-\mathrm{NMR},{ }^{13} \mathrm{C}$ NMR, Mass) and data CV of all the molecules reported in the experimental section are included in the Supporting Information.

\section{- ACKNOWLEDGMENTS}

The authors would like to thank any person who helped in the realization of this work, as well as the team of the laboratory of the Organic Chemistry Department of the University Alicante.

\section{- REFERENCES}

[1] Akamatsu, H., Inokuchi, H., and Matsunaga, Y., 1954, Electrical conductivity of the perylenebromine complex, Nature, 173 (4395), 168-169.

[2] Saito, G., and Yoshida, Y., 2012, Frontiers of organic conductors and superconductors, Top. Curr. Chem., 312, 67-126.

[3] Martin, N., 2013, Tetrathiafulvalene: The advent of organic metals, Chem. Commun., 49 (63), 70257027. 
[4] Filatre-Furcate, A., Higashino, T., Lorcy, D., and Mori, T., 2015, Air-stable n-channel organic fieldeffect transistors based on a sulfur rich $\pi$-electron acceptor, J. Mater. Chem. C, 3 (15), 3569-3573.

[5] Gal-Oz, R., Patil, N., Khalfin, R., Cohen, Y., and Zussman, E., 2013, Conductive PVDF-HFP nanofibers with embedded TTF-TCNQ charge transfer complex, ACS Appl. Mater. Interfaces, 5 (13), 6066-6072.

[6] Prokhorova, T.G., and Yagubskii, E.B., 2017, Organic conductors and superconductors based on bis(ethylenedithio)tetrathiafulvalene radical cation salts with supramolecular tris(oxalato)metallate anions, Russ. Chem. Rev., 86 (2), 164.

[7] Paxton, W.F., Kleinman, S.L., Basuray, A.N., Stoddart, J.F., and Van Duyne, R.P., 2011, Surfaceenhanced Raman spectroelectrochemistry of TTFmodified self-assembled monolayers, J. Phys. Chem. Lett., 2 (10), 1145-1149.

[8] Pérez-Rentero, S., Eritja, R., Häring, M., Saldías, C., and Díaz, D.D., 2018, Synthesis, characterization, and self-assembly of a tetrathiafulvalene (TTF)triglycyl derivative, Appl. Sci., 8 (5), 671.

[9] Nair, M.N., Mattioli, C., Cranney, M., Malval, J.P., Vonau, F., Aubel, D., Bubendorff, J.L., Gourdon, A., and Simon, L., 2015, STM studies of self-assembled tetrathiafulvalene (TTF) derivatives on graphene: Influence of the mode of deposition, J. Phys. Chem. C, 119, 9334-9341.

[10] Tian, J., Ding, Y.D., Zhou, T.Y., Zhang, K.D., Zhao, X., Wang, H., Zhang, D.W., Liu, Yi., and Li, Z.T., 2014, Self-assembly of three-dimensional supramolecular polymers through cooperative tetrathiafulvalene radical cation dimerization, Chem. Eur. J., 20 (2), 575-584.

[11] Jain, A., Rao, K.V., Mogera, U., Sagade, A.J., and George, S., 2011, Dynamic self-assembly of chargetransfer nanofibers of tetrathiafulvalene derivatives with F 4 TCNQ, Chem. Eur. J., 17 (44), 12355-12361.

[12] Evans, N.H., Rahman, H., Davis, J.J., and Beer, P.D., 2012, Surface-attached sensors for cation and anion recognition, Anal. Bioanal. Chem., 402 (5), 17391748 .
[13] Zhao, B.T., Cao, S.N., Guo, H.M., and Qu, G.R., 2013, Metal-ion-promoted intermolecular electron transfer between anthraquinone-based tetrathiafulvalene derivative and $p$-chloranil, Synth. Met., 174, 14-18.

[14] Shao, M., Dongare, P., Dawe, L.N., Thompson, D.W., and Zhao, Y., 2010, Biscrown-annulated TTFAQ-dianthracene hybrid: Synthesis, structure, and metal ion sensing, Org. Lett., 12 (13), 30503053.

[15] Blanchard, P.Y., Alévêque, O., Boisard, S., Gautier, C., El-Ghayoury, A., Le Derf, F., Breton, T., and Levillain, E., 2011, Intermolecular interactions in self-assembled monolayers of tetrathiafulvalene derivatives, Phys. Chem. Chem. Phys., 13 (6), 21182120.

[16] Yuge, R., Miyazaki, A., Enoki, T., Tamada, K., Nakamura, F., and Hara, M., 2002, Electrochemical properties of self-assembled monolayers composed of TTF derivative, Mol. Cryst. Liq. Cryst., 377 (1), 395-398.

[17] Gomar-Nadal, E., Ramachandran, G.K., Chen, F., Burgin, T., Rovira, C., Amabilino, D.B., and Lindsay, S.M., 2004, Self-assembled monolayers of tetrathiafulvalene derivatives on $\mathrm{Au}(111)$ : Organization and electrical properties, J. Phys. Chem. B, 108 (22), 7213-7218.

[18] Yokota, Y., Yuge, R., Miyazaki, A., Enoki, T., and Hara, M., 2003, Property of self-assembled monolayers of long-alkyl-chain-substituted TTF dirivative, Mol. Cryst. Liq. Cryst., 407 (1), 121-127.

[19] Yuge, R., Miyazaki, A., Enoki, T., Ito, E., Nakamura, F., and Hara, M., 2001, Characterization and electronic properties of TTF SAMs on $\mathrm{Au}(111)$, Mol. Cryst. Liq. Cryst. Sci. Technol., Sect. A, 370 (1), 273-276.

[20] Suzuki, A., Inoue, K., Yano, K., Oku, T., and Kikuchi, K., 2010, Fabrication and characterization of $\mathrm{C}_{60} /$ tetrathiafulvalene solar cells, J. Phys. Chem. Solids, 71 (11), 1587-1591.

[21] Martín, N., Sánchez, L., Herranz, M.Á., Illescas, B., and Guldi, D.M., 2007, Electronic communication in tetrathiafulvalene $(\mathrm{TTF}) / \mathrm{C}_{60}$ systems: Toward 
molecular solar energy conversion materials, Acc. Chem. Res., 40 (10), 1015-1024.

[22] Martin-Gomis, L., Nielsen, K.A., Fernandez-Lázaro, F., Jeppesen, J.O., and Sastre-Santos, A., 2011, Supramolecular (2,5,7-trinitrofluorene)- $\mathrm{C}_{60} /$ tetrathia fulvalene-calix[4]pyrrole systems, Meet. Abstr., 01, 1692.

[23] Konarev, D.V., Shul'ga, Y.M., Roshchupkina, O.S., and Lyubovskaya, R.N., 1997, Synthesis and some properties of charge transfer complexes of $\mathrm{C}_{60}$ with asymmetric donors of tetrathiafulvalene family, $J$. Phys. Chem. Solids, 58 (11), 1869-1872.

[24] Lai, G., Liu, Y., Zhang, Y., Ruan, J., Li, M., and Shen, Y., 2009, Synthesis and properties of tetrathiafulvalene-fluorescein dyads, Front. Chem. Eng. China, 3 (3), 314-317.

[25] Mas-Torrent, M., Hadley, P., Bromley, S.T., Ribas, X., Tarrés, J., Mas, M., Molins, E., Veciana, J., and Rovira, C., 2004, Correlation between crystal structure and mobility in organic field-effect transistors based on single crystals of tetrathiafulvalene derivatives, $J$. Am. Chem. Soc., 126 (27), 8546-8553.

[26] Schröder, H.V., and Schalley, C.A., 2018, Tetrathiafulvalene - A redox-switchable building block to control motion in mechanically interlocked molecules, Beilstein J. Org. Chem., 14, 2163-2185.

[27] Wang, C., Dyar, S.M., Cao, D.C., Fahrenbach, A.C., Horwitz, N., Colvin, M.T., Carmieli, R., Stern, C.L., Dey, S.K., Wasielewski, M.R., and Stoddart, J.F., 2012, Tetrathiafulvalene hetero radical cation dimerization in a redox-active [2]catenane, J. Am. Chem. Soc., 134 (46), 19136-19145.

[28] Pauliukaite, R., Malinauskas, A., Zhylyak, G., and Spichiger-Keller, U.E., 2007, Conductive organic complex salt TTF-TCNQ as a mediator for biosensors. An overview, Electroanalysis, 19 (24), 2491-2498.

[29] Cao, Z., Jiang, X., Xie, Q., and Yao, S., 2008, A thirdgeneration hydrogen peroxide biosensor based on horseradish peroxidase immobilized in a tetrathiafulvalene-tetracyanoquinodimethane/multi walled carbon nanotubes film, Biosens. Bioelectron., 24 (2), 222-227.
[30] Tehfe, M.A., Monot, J., Malacria, M., Fensterbank, L., Fouassier, J.P., Curran, D.P., Lacôte, E., and Lalevée, J., 2012, A water-compatible NHC-borane: Photopolymerizations in water and rate constants for elementary radical reactions, ACS Macro Lett., 1 (1), 92-95.

[31] Zappe, L., Schönfeld, S., Hörner, G., Zenere, K.A., Leong, C.F., Kepert, C.J., D'Alessandro, D.M., Weber, B., and Neville, S.M., 2020, Spin crossover modulation in a coordination polymer with the redox-active bis-pyridyltetrathiafulvalene ( $\left.\mathrm{py}_{2} \mathrm{TTF}\right)$ ligand, Chem. Commun., 56 (72), 10469-10472.

[32] Wang, H.Y., Cui, L., Xie, J.Z., Leong, C.F., D'Alessandro, D.M., and Zuo, J.L., 2017, Functional coordination polymers based on redox-active tetrathiafulvalene and its derivatives, Coord. Chem. Rev., 345, 342-361.

[33] Nambu, S., Nakahodo, T., and Fujihara, H., 2014, Synthesis and properties of conducting polymer nanotubes with redox-active tetrathiafulvalene, Heterocycles, 88 (2), 1633-1638.

[34] Nielsen, M.B., Lomholt, C., and Becher, J., 2000, Tetrathiafulvalenes as building blocks in supramolecular chemistry II, Chem. Soc. Rev., 29 (3), 153-164.

[35] Miyasaka, H., Motokawa, N., Matsunaga, S., Yamashita, M., Sugimoto, K., Mori, T., Toyota, N., and Dunbar, K.R., 2010, Control of charge transfer in a series of $\mathrm{Ru}_{2}{ }^{\mathrm{II}, \mathrm{II}} / \mathrm{TCNQ}$ two-dimensional networks by tuning the electron affinity of TCNQ units: A route to synergistic magnetic/conducting materials, J. Am. Chem. Soc., 132 (5), 1532-1544.

[36] Guérin, D., Carlier, R., Guerro, M., and Lorcy, D., 2003, Crown-ether annelated dithiadiazafulvalenes, Tetrahedron, 59 (28), 5273-5278.

[37] Singh, S.P., Parmar, S.S., Raman, K., and Stenberg, V.I., 1981, Chemistry and biological activity of thiazolidinedinones, Chem. Rev., 81 (2), 175-203.

[38] Mahalle, S.R., Netankar, P.D., Bondge, S.P., and Mane, R.A., 2008, An efficient method for Knoevenagel condensation: a facile synthesis of 5arylidenyl 2, 4-thiazolidinedione, Green Chem. Lett. Rev., 1 (2), 103-106. 
[39] Guérin, D., 2001, Modulation des propriétés rédox $\mathrm{du}$ donneur $\pi$ dithiadiazafulvalène appliquée à la formation de matériaux moléculaires, Dissertation, Institut des Sciences Chimiques de Rennes (ISCR), Université de Rennes 1, Rennes, France.

[40] Broggi, J., Terme, T., and Vanelle, P., 2014, Organic electron donors as powerful single-electron reducing agents in organic synthesis, Angew. Chem. Int. Ed., 53 (2), 384-413.
[41] Janikowska, K., and Makowiec, S., 2010, Simple method for the preparation of dialkyl (2,3-dihydro1,3-thiazol-2-YL)-phosphonates, Phosphorus, Sulfur Silicon Relat. Elem., 186 (1), 12-20.

[42] Časar, Z., Leban, I., Majcen-le Marechal, A., Piekara-Sady, L., and Lorcy, D., 2009, Charge transfer complexes and cation radical salts of azinodiselenadiazafulvalene, C.R. Chim., 12 (9), 10571065. 